\title{
Functional design of bacterial superoxide:quinone oxidoreductase
}

Abbas Abou-Hamdan+1, Roman Mahlert¹, Philipp Grossenbacher², Olivier Biner ${ }^{1,3}$, Dan Sjöstrand ${ }^{4}$, Martin Lochner ${ }^{2}$, Martin Högbom ${ }^{4}$ and Christoph von Ballmoos ${ }^{1, *}$

\section{Affiliations:}

${ }^{1}$ Department of Chemistry, Biochemistry and Pharmaceutical Sciences, University of Bern, 3012 Bern, Switzerland.

${ }^{2}$ Institute of Biochemistry and Molecular Medicine, University of Bern, 3012 Bern, Switzerland

${ }^{3}$ Department of Plant and Microbial Biology, University of Zürich, 8008 Zürich, Switzerland

${ }^{4}$ Stockholm center for Biomembrane Research, Department of Biochemistry and Biophysics, Stockholm University, 10691 Stockholm, Sweden.

*Correspondence to: christoph.vonballmoos@unibe.ch

+ Contributed equally 


\section{Abstract}

The superoxide anion - molecular oxygen reduced by a single electron - is produced in large amounts by enzymatic and adventitious reactions and can perform a range of cellular functions, including bacterial warfare and iron uptake, signalling and host immune response in eukaryotes. However, it also serves as precursor for more deleterious species such as the hydroxyl anion or peroxynitrite and therefore, cellular defense mechanisms for superoxide neutralization have evolved. In addition to the soluble proteins superoxide dismutase and superoxide reductase, recently the membrane embedded diheme cytochrome $b_{561}$ (CybB) from $E$. coli has been proposed to act as a superoxide:quinone oxidoreductase. Here, we confirm superoxide and cellular ubiquinones or menaquinones as natural substrates and show that quinone binding to the enzyme accelerates the reaction with superoxide. The reactivity of the substrates is in accordance with the here determined midpoint potential of the two $b$ hemes (+48 and -23 mV / NHE). Our data suggest that the enzyme can work near the diffusion limit in the forward direction and can also catalyse the reverse reaction efficiently under physiological conditions. The data is discussed in context of described cytochrome $b_{561}$ proteins and potential physiological roles of CybB. 


\section{Introduction}

In all aerobic organisms, oxygen plays a vital role in the conversion of reducing equivalents into the universal cellular energy currency ATP. During the last step of respiration, terminal oxidases catalyze the highly exergonic reduction of molecular oxygen to water. This reaction is coupled to the formation of a transmembrane electrochemical proton gradient (or proton motive force, $p m f$ ), which is harnessed by the ATP synthase to recycle ATP from ADP and inorganic phosphate. In contrast to this well controlled reaction, adventitious single electron transfer to molecular oxygen results in formation of reactive oxygen species (ROS), representing incompletely reduced oxygen variants such as superoxide $\left(\mathrm{O}_{2}{ }^{-}\right)$, hydrogen peroxide $\left(\mathrm{H}_{2} \mathrm{O}_{2}\right)$ and hydroxyl radical $\left(\mathrm{OH}^{-}\right)$. Depending on their concentration, ROS can either be toxic or function as signalling molecules, playing important roles in normal physiological and pathophysiological processes ${ }^{1}$. High and sustained concentrations of ROS are known to have deleterious effects on cellular health by oxidizing the three major biomolecule classes DNA, proteins, and lipids ${ }^{2}$. As molecular oxygen preferably partitions into the lipophilic environment of the membrane, the electron transfer reactions of the membrane embedded respiratory chain enzymes are a prominent source of ROS, e.g. at the flavin sites of complex $\mathrm{I}^{3}$ and $\mathrm{II}^{4}$ and the ubiquinol oxidation center of complex $\mathrm{II}^{5}$. Single electron transfer to molecular oxygen yields superoxide, which shows relatively mild deleterious cellular effects, but has been shown to directly damage the metal centers of enzymes ${ }^{6}$. In eukaryotes, superoxide is actively produced by the enzyme NAPDH oxidase as a second messenger, but also to serve as a first line of defense against bacterial infections ${ }^{7-9}$. In prokaryotes, extracellular superoxide production has been observed in marine bacteria to support metal uptake $^{10}$, and as well as in Enterococcus faecalis, E. coli and Vibrio cholerae ${ }^{11-13}$. The main defense mechanism is production of superoxide dismutase, first described in 1969, which greatly accelerates the disproportionation of two superoxide molecules to one molecule of hydrogen peroxide and one molecule of oxygen ${ }^{14,15}$. Some twenty years later, a second enzyme, superoxide reductase, was described in anaerobic bacteria to react specifically with superoxide, forming hydrogen peroxide using reduced rubredoxin as electron donor ${ }^{16}$.

Yet another 20 years later, in 2018, our groups described a third, membrane-embedded enzyme, encoded by the Escherichia coli gene $c y b B$ with the gene product known as cytochrome $b_{561}(\mathrm{CybB})$. In contrast to the two other enzymes, CybB catalyzes the oxidation 
of superoxide to oxygen and transfers the extracted electron to the oxidized quinone pool in the membrane ${ }^{17}$ (Fig. 1A). Therefore, CybB is a superoxide:ubiquinone oxidoreductase or, in short, superoxide oxidase (SOO). The crystal structure and functional data suggested that in the forward reaction, superoxide binds to a patch of positively charged amino acid residues at the periplasmic surface. This triggers a transmembrane electron transfer reaction via two $b$-type hemes, and the reduction of ubiquinone bound near the cytoplasmic surface of the protein (Fig. 1A). The two hemes show a close edge-to-edge distance of $11 \AA$ allowing a fast electron transfer ${ }^{18,19}$. When CybB was mixed with reduced ubiquinol, the reverse reaction, i.e., reduction of CybB hemes and production of superoxide, was observed. The direction of catalysis under physiological conditions and the metabolic role of CybB are unknown. However, $m R N A$ levels of $c y b B$ were found to be more than 10-times higher in the exponential than in the stationary phase whilst being unaffected by the presence of oxygen ${ }^{17}$.

Here, we thoroughly characterize detergent-solubilised superoxide:quinone oxidoreductase from E. coli using a range of biochemical and biophysical techniques. We provide evidence that superoxide and cellular quinone are indeed its natural substrate and measure the $\mathrm{pH}$ dependence of the reverse reaction. The data are then interpreted in connection to the heme mid-point potential (also determined in this study) in the absence and presence of quinone. Using a coupled enzymatic assay, we determine that the apparent affinity constant $K_{\mathrm{m}}$ for quinones is in the submicromolar range. 


\section{Materials and methods}

\section{Expression of CybB}

Expression of CybB was done as published previously ${ }^{17}$. Briefly, the plasmid pcybB-His encoding for the E. coli $c y b B$ gene with an 8xHis-tag fusion at its C-terminus was used to transform chemically competent $E$. coli BL21(DE3) pLysS cells. Transformed bacteria were grown on LB-agar plates containing $30 \mathrm{\mu g} \mathrm{ml}^{-1}$ kanamycin overnight at $37^{\circ} \mathrm{C}$. One colony was picked to inoculate a culture in $100 \mathrm{ml}$ LB media containing kanamycin $\left(30 \mu \mathrm{g} \mathrm{ml}^{-1}\right)$. Large culture growth was conducted at $37{ }^{\circ} \mathrm{C}$ using a LeX48 system (Epiphyte 3, Canada) where conventional shaking was replaced by continuous air bubbling. At a culture absorbance $\left(\mathrm{OD}_{600}\right)$ between 0.9 and 1.1, expression of $\mathrm{CybB}$ was induced by the addition of $0.2 \mathrm{mM}$ IPTG. Cells were harvested 4 hours after induction by centrifugation at $7500 \times \mathrm{g}$ and the pellet was resuspended in buffer (50 mM Hepes pH 7.5, $200 \mathrm{mM} \mathrm{NaCl}$ and $7.5 \%$ glycerol).

\section{Membrane preparation and protein purification}

To prepare membranes, the resuspended pellet was mixed with $1 \mathrm{mM} \mathrm{PMSF}, 2 \mathrm{mM} \mathrm{MgCl}$, some crystals of PEFA-bloc (Biomol, Germany), lysozyme and DNase I (Merck, Germany). The mixture was stirred for $30 \mathrm{~min}$ at RT and the homogenized cells were broken at least twice at 2000 Bar utilizing a Maximator High Pressure Homogenizer HPL6 (Maximator AG, Switzerland). Unbroken cells were removed by centrifugation at $7500 \times \mathrm{g}$ for $15 \mathrm{~min}$ and the supernatant transferred to a Beckman Ti45 ultracentrifuge rotor and centrifuged at 40000 rpm for $45 \mathrm{~min}$. The membrane pellet was resuspended in the resuspension buffer and diluted up to $10 \mathrm{mg} \mathrm{ml}^{-1}$. Diluted membranes were solubilized by the addition of $1 \%$ of OGNG (or 1 \% DDM) (Anatrace) in the presence of $1 \mathrm{mM}$ PMSF and some crystals of PEFA-Bloc for $1 \mathrm{~h}$ at $4{ }^{\circ} \mathrm{C}$. Insoluble material was cleared by ultracentrifugation for $45 \mathrm{~min}$ at $40000 \mathrm{rpm}$ in a Beckman Ti70 rotor. The resulting supernatant was loaded onto the nickel-charged Profinity IMAC Resin (Bio-Rad, USA) and washed with 15 column volumes (CV) of wash buffer (10 mM Hepes pH 7.5, $200 \mathrm{mM} \mathrm{NaCl}, 7.5 \%$ glycerol and $0.1 \%$ OGNG) and $15 \mathrm{CV}$ of wash buffer 2 (10 mM Hepes pH 7.5, 200 mM NaCl, $7.5 \%$ glycerol, $0.1 \%$ OGNG and 5 mM Histidine). CybB was eluted with 10CV of elution buffer (10 mM Hepes pH 7.5, $200 \mathrm{mM} \mathrm{NaCl}, 7.5 \%$ glycerol, $0.1 \%$ OGNG containing either $200 \mathrm{mM}$ histidine, $100 \mathrm{mM}$ EDTA or $200 \mathrm{mM}$ Imidazole) and concentrated up to $0.5 \mathrm{ml}$ using Amicon Ultra-15 centrifugal filters with MWCO of $50 \mathrm{kDa}$ (Merck, Germany). The sample was then applied to a size exclusion chromatography on a 
Superdex 200 increase 10 /300GL column equilibrated with wash buffer. Fractions containing CybB were pooled together, concentrated with a MWCO 50 kDa concentrator, and flashfrozen in liquid nitrogen.

\section{Reduction of CybB}

Reduction of CybB was monitored using a Cary 60 UV-Vis Spectrophotometer (Agilent Technologies, USA) by measuring heme absorbance changes at either $428 \mathrm{~nm}$ or $561 \mathrm{~nm}$ upon addition of various substrates in aerobic conditions. All measurement were done in $100 \mathrm{mM}$ sodium phosphate $\mathrm{pH} 8,0.1 \mathrm{mM}$ diethylenetriaminepentaacetic acid (DTPA) and $0.05 \%$ DDM in the presence or absence of $0.1 \mathrm{mM}$ hypoxanthine (HPX) depending on whether superoxide is produced by xanthine oxidase (XO) or not. In those measurements, 0.25 to $1 \mu \mathrm{M}$ of CybB was used and the reaction started by addition of superoxide (generated enzymatically by using $0.01 \mathrm{U}$ xanthine oxidase from bovine milk (Sigma, USA) that catalyse the oxidation of hypoxanthine to xanthine and / or xanthine to uric acid and produces superoxide as byproduct, chemically by dissolving $7.1 \mathrm{mg} \mathrm{KO} 2$ in $1 \mathrm{ml}$ of anhydrous DMSO in the presence of $80 \mathrm{mg}$ of 18-crown-6 ether to stabilize produced superoxide under steam of $\mathrm{N}_{2}{ }^{20}$ or upon using of $1 \mathrm{mM} \mathrm{NADH} \mathrm{/} 10 \mu \mathrm{MPMS}^{21}$ ) or other reductants such as $1 \mathrm{mM}$ dithiothreitol (DTT) and 1 $\mathrm{mM}$ ascorbate. For control measurements, $8.8 \mu \mathrm{g} \mathrm{ml}^{-1}$ of superoxide dismutase (Sigma) was used to disproportionate superoxide anion in the presence of $20 \mu \mathrm{g} \mathrm{ml}^{-1}$ of catalase (Sigma). Full reduction of CybB was achieved upon addition of sodium dithionite and used hence to calculate the percentage of relative heme reduction. Ubiquinone analogues were either synthetized $\left(Q_{1}, Q_{2}\right.$ and decylubiquinone) as described in the supplementary material or purchased from Sigma. Ubiquinone analogues $\left(Q_{1}, Q_{2}\right.$ and decylubiquinone) and naphthalene analogues (1,4 naphthoquinone and menadione, a soluble form of menaquinone) were reduced by incubation of $50 \mathrm{mM}$ quinone solution with $1 \mathrm{M}$ DTT with respect of $\mathrm{V}_{\mathrm{DTT}} / \mathrm{V}_{\mathrm{Q}}$ ratio of 4 to ensure a complete reduction of quinone or pre-reduced with sodium borohydride $\mathrm{NaBH}_{4}$ (as with $\mathrm{Q}_{2}$ ). Reduced quinones were hereafter added to reduce $\mathrm{CybB}$ and the percentage of relative quinol heme reduction was calculated as mentioned previously.

For the $\mathrm{pH}$ dependency measurements, we used the same protocol as before and measured either superoxide or $\mathrm{Q}_{1} \mathrm{H}_{2}$ induced heme reduction in the presence of different buffered solutions. In the first set of experiment, we used $100 \mathrm{mM}$ sodium phosphate ( $\mathrm{pH} 7$ or 8 ) or $100 \mathrm{mM}$ CHES and $100 \mathrm{mM}$ sodium phosphate dibasic (pH 9 or 10) containing $20 \mathrm{mM} \mathrm{KCl}, 200$ 
$\mathrm{mM} \mathrm{NaCl}, 0.1 \mathrm{mM}$ DPTA, $0.05 \%$ DDM. In the second set, we used $100 \mathrm{mM}$ sodium phosphate (for $\mathrm{pH}$ ranging from 6 to 8.5) or $20 \mathrm{mM}$ Tris- $\mathrm{HCl}$ (for $\mathrm{pH}$ ranging from 9 to 10) containing each $20 \mathrm{mM} \mathrm{KCl}, 200 \mathrm{mM} \mathrm{NaCl}, 0.1 \mathrm{mM}$ DPTA, $0.05 \%$ DDM. In both experiments, the percentage of relative heme reduction compared to a full dithionite reduction of CybB was calculated. Anaerobic measurements were performed after removal of dioxygen using a combined system of argon blowing in the presence of $53 \mu \mathrm{g} \mathrm{ml}^{-1}$ catalase, $26 \mathrm{\mu g} \mathrm{ml}^{-1}$ glucose oxidase and $5 \mathrm{mM}$ glucose mixture (all purchased from Sigma).

\section{Oxidation of reduced CybB}

Oxidation of reduced CybB was monitored under anaerobic condition. Anaerobicity was achieved as described above (section: Reduction of $C y b B$ ). 15 min after argon blowing, 0.25 $\mu \mathrm{M}$ of CybB was fully pre-reduced using a tiny amount of dithionite $(6.6 \mu \mathrm{M}$ in $1.5 \mathrm{ml}$ of 100 $\mathrm{mM}$ sodium phosphate $\mathrm{pH} 8,20 \mathrm{mM} \mathrm{KCl}, 200 \mathrm{mM} \mathrm{NaCl}, 0.05 \% \mathrm{DDM})$ before addition of 10 $\mu \mathrm{M}$ of diverse quinone (ubiquinone and naphthalene analogue) to oxidize it. A full oxidation of reduced CybB was achieved by adding $10 \mu \mathrm{M}$ of potassium ferricyanide and used to calculate the percentage of relative heme oxidation.

\section{Superoxide assay and $K_{m}$ determination}

We used the previously established WST-1 (Water soluble Tetrazolium dye) assay ${ }^{22}$ and adapted it ${ }^{17}$ to determine either the apparent affinity constant for ubiquinone of CybB or to measure superoxide production by the enzyme. In both assays, formazan formation upon reduction of WST-1 by superoxide was monitored at $438 \mathrm{~nm}$ in assay buffer (100 mM sodium phosphate pH 8, 0.1 mM DTPA, $0.1 \mathrm{mM} \mathrm{HPX}$, and 0.05 \% DDM) containing $50 \mu \mathrm{M}$ WST-1, 20 $\mu \mathrm{g} \mathrm{ml}^{-1}$ catalase and $60 \mathrm{nM}$ bo3 oxidase. Measurements were done either individually in a cuvette using Cary 60 UV-Vis spectrophotometer or in a 96-well plate in SpectraMax ABS Plus Microplate Reader (Molecular Devices, USA). Ubiquinone $\mathrm{Q}_{1}$ or $\mathrm{Q}_{2}$ were added to the previous mixture to measure the overall activity of CybB. The apparent Michaelis Menten constant $\left(K_{m}\right)$ for quinone was calculated using a constant amount of CybB (50 nM) while varying the quinone concentration between 0 and $100 \mu \mathrm{M}$. To measure superoxide production and determine the apparent constant affinity for quinol, the reaction was initiated by addition of $50 \mathrm{nM}$ of CybB to the assay buffer containing different concentration of quinol (0 - $100 \mu \mathrm{M})$ in the absence of $b o_{3}$ oxidase and catalase. 
The second order rate (catalytic efficiency) of CybB was calculated based on the known value for SOD $\left(2 \times 10^{9} \mathrm{M}^{-1} \mathrm{~s}^{-1}\right)^{23}$. Briefly, the concentration for both enzymes SOD and CybB was titrated using the former assay to reduce the formazan formation rate by $50 \%$. The determined concentrations were used to calculate the catalytic efficiency of CybB.

\section{Potentiometric redox titrations}

Redox titrations were performed anaerobically at room temperature in a Spectroelectrochemical cell (Prosense, Netherlands), by following heme absorption changes upon a gradual addition of dithionite solution as electron source, instead of electrochemistry as in ${ }^{24}$ using a UV-Vis spectrophotometer.

Throughout the measurement, anaerobicity was maintained by a constant and continuous flow of argon. Prior to measuring, $10 \mu \mathrm{M}$ CybB, in the absence or presence of $20 \mu \mathrm{M} \mathrm{Q}_{1}$, and $5 \mu \mathrm{M}$ mediators were incubated together in $100 \mathrm{mM}$ sodium phosphate $\mathrm{pH}$ 7, $0.1 \mathrm{mM}$ DTPA, $0.1 \mathrm{mM}$ HPX, and $0.05 \% \mathrm{DDM}$ and flushed with a repetitive and alternative cycles of $\mathrm{N}_{2}$ / vacuum to ensure complete removal of oxygen. The following mediators were used: benzyl viologen (-350 $\mathrm{mV})$, sodium anthraquinone 2-sulfonate $(-225 \mathrm{mV})$, 2-hydroxy 1,4naphtoquinone $(-145 \mathrm{mV})$, resorufin $(-51 \mathrm{mV})$, methylene blue $(+11 \mathrm{mV})$, phenazine ethosulfate $(+55 \mathrm{mV})$, phenazine methosulfate $(+80 \mathrm{mV}), 2,6$-Dichlorophenol-indophenol (+217 mV) and tetramethyl-p-phenylendediamine (+276 mV). Redox potentials were measured using an $\mathrm{Ag} / \mathrm{AgCl} 3 \mathrm{M} \mathrm{KCl}$ reference electrode associated to a platinum counter electrode (Pt) (Prosense, Netherlands). The redox potential was noted after equilibration, and an optical spectrum was recorded between $400-600 \mathrm{~nm}$. The measured absorbance of heme reduction at $561 \mathrm{~nm}$ was plotted against the measured redox potentials and curves were fitted using the Nernst equation with $n=2$ due to two successive one electron reductions corresponding to the reduction of both hemes. All midpoint potentials referenced here were adjusted by the addition of $+210 \mathrm{mV}$ relative to the normal hydrogen electrode (VS NHE) at $25^{\circ} \mathrm{C}$. 


\section{Results}

\section{Enzyme production and purification}

The gene sequence of $E$. coli $c y b B$, equipped with a C-terminal octahistidine tag was cloned into a pET-28b vector under control of the lac promoter. As a host strain, BL21(DE3) pLysS was used. The protein is well expressed yielding cells and inverted membranes with a distinct pink color. Here, we further optimized the purification of CybB based on our published protocol ${ }^{17}$. The membrane fraction was solubilized with either $1 \%$ OGNG or $1 \%$ DDM and purified via Ni-IDA chromatography. The protein was eluted using either imidazole, histidine or EDTA, concentrated and assessed using size-exclusion chromatography (Superdex 200 Increase, $10 / 300 \mathrm{GL}, 500 \mu \mathrm{l}$ injected), using either $0.1 \%$ OGNG or $0.1 \%$ DDM. The results displayed in supplementary Figure S1 show a major impact of both the detergent and the $\mathrm{Ni}$ IDA eluant used. While both detergents extracted the protein similarly from the membrane, only extractions with OGNG produced a monomeric peak ( $14.7 \mathrm{ml})$, as judged from the size exclusion chromatograms (Fig. S1 A and E). In addition to this peak (Fig S1A), two further discrete elution peaks were observed, indicating aggregate forms of the protein. If the protein was eluted with either 100 mM EDTA or 200 mM histidine (Fig. S1 A and E) instead of imidazole a homogenous preparation with a single monomeric peak was obtained. In the following, $1 \%$ OGNG and $200 \mathrm{mM}$ histidine in $0.1 \%$ OGNG was used for solubilization and elution, respectively.

\section{Spectroscopic properties and reaction with cellular reductants}

The crystal structure of CybB shows a highly distorted 4-helix bundle with the $b$-hemes fully coordinated by histidines (Fig. 1B). The protein is almost entirely in contact with the lipid bilayer with no peripheral domains. Heme 2 is partly exposed towards the periplasm by a narrow cavity that contains a variety of positively charged residues that are proposed to guide the negatively charged superoxide anion in proximity of the heme to allow electron transfer. Heme 1 is located towards the cytoplasm and buried in the membrane with no polar access route, supporting the idea of a binding site for a hydrophobic compound such as ubiquinone. The two hemes are rapidly reduced by dithionite and the reduced spectrum shows a characteristic Soret peak at $428 \mathrm{~nm}$ as well as $\alpha$ and $\beta$ peaks at 530 and $561 \mathrm{~nm}$ as described earlier. However, all other attempts to fully reduce the enzyme with cellular reductants or ubiquinols failed. Lundgren et $\mathrm{al}^{17}$. found that detergent solubilized CybB is rapidly, but only 
bioRxiv preprint doi: https://doi org/10.1101/2021.12 23.473985; this version posted December 23, 2021. The copyright holder for this preprint (which was not certified by peer review) is the author/funder, who has granted bioRxiv a license to display the preprint in perpetuity. It is made available under aCC-BY-ND 4.0 International license.

partly reduced ( $50 \%$ ) by the DTT-reduced short-chain ubiquinone analogue $\mathrm{Q}_{1}$. Partial reduction by superoxide was also only observed when using xanthine oxidase as superoxide producing system. No or only very slow reduction is observed with reducing agents such as ascorbate, DTT and NADH (Fig. 1C). We therefore aimed to better understand the reduction behaviour of detergent solubilized CybB in a series of kinetic and steady state experiments.

A

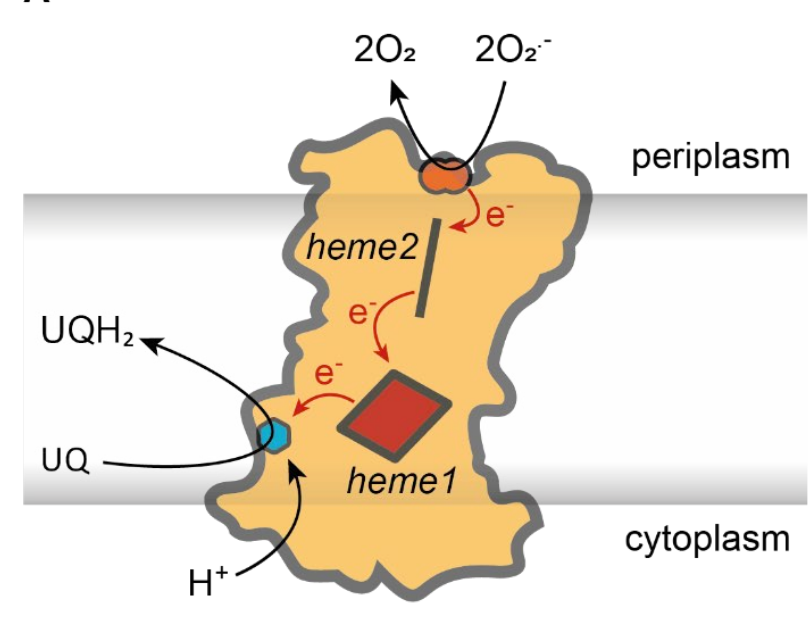

C

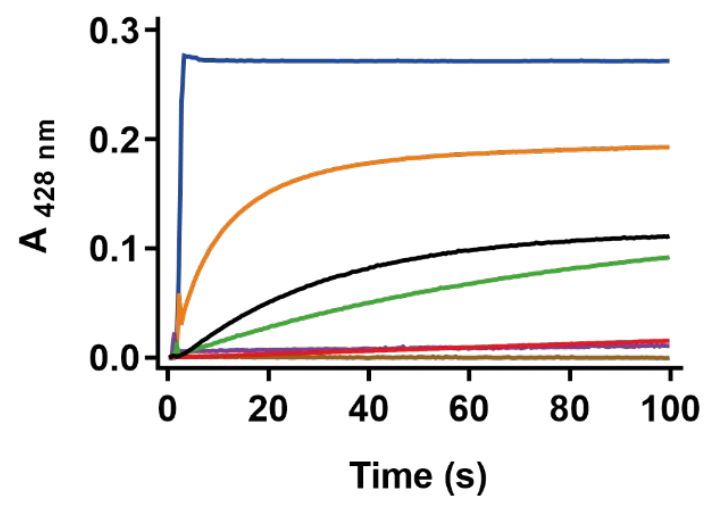

B

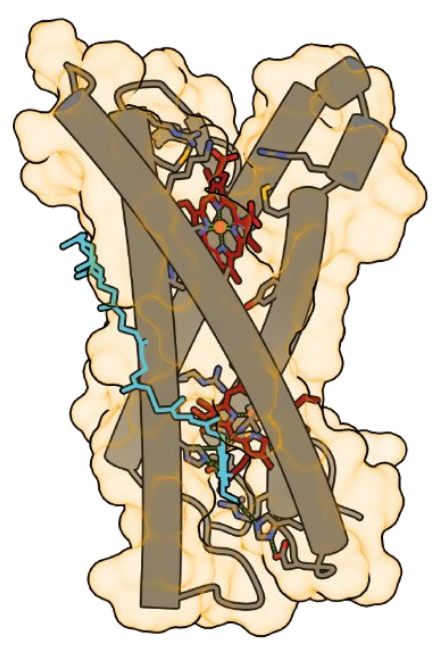

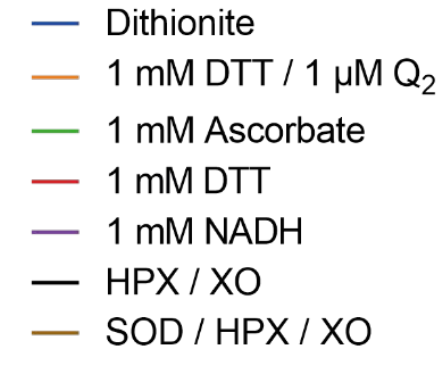

Figure 1: CybB structure and the corresponding substrates. A) Proposed reaction mechanism of CybB. Upon oxidation of superoxide anion at the heme 2, electrons are transferred through the two hemes to reduce the membrane quinone pool with protons being sequestrated from the cytoplasm B) Distorted helical arrangement of CybB produced by ECSF Chimera X. The 4 alpha helices are shown in grey, heme 2 and heme1 in dark red and ubiquinone UQ8 in cyan. Visualization of ubiquinone $Q_{8}$ (PubChem CID 5283546) as suggested by molecular docking to the crystal structure (pdb 50C0) using AutoDock Vina ${ }^{25}$. C) Kinetic traces monitoring the CybB reduction at $428 \mathrm{~nm}$ upon addition of different substrates: $1 \mathrm{mM}$ sodium dithionite (blue), $1 \mathrm{mM}$ dithiothreitol (DTT) (red), $1 \mathrm{mM} \mathrm{DTT} \mathrm{/} 1$ uM ubiquinone 2 ( $\mathrm{Q}_{2}$ ) (orange), $0.1 \mathrm{mM}$ Hypoxanthine / $7.6 \mathrm{mU}$ Xanthine oxidase (HPX / XO) (black), $1 \mathrm{mM}$ Ascorbate (green) and $1 \mathrm{mM} \mathrm{NADH}$ (purple). Measurements were done in $100 \mathrm{mM}$ sodium phosphate $\mathrm{pH} 8,0.1 \mathrm{mM}$ DTPA, $0.05 \%$ DDM. When xanthine oxidase is used, $0.1 \mathrm{mM}$ of hypoxanthine was added to the previous buffer. $8.8 \mathrm{\mu g} \mathrm{ml}^{-1}$ of SOD was present before addition of substrates (brown). 
Reaction of $\mathrm{Cy} b \mathrm{~B}$ with superoxide produced by $\mathrm{KO}_{2}$ and NADH / PMS

Superoxide in aqueous solution has a half-life that is strongly $\mathrm{pH}$ dependent, as the spontaneous disproportionation to hydrogen peroxide and oxygen occurs via the protonated $\mathrm{O}_{2} \mathrm{H}$ form. Consequently, superoxide is more stable at higher $\mathrm{pH}$ values, i.e., $\mathrm{t} \frac{1}{2} \sim 1 \mathrm{~s}$ at $10 \mu \mathrm{M}$ and $\mathrm{pH}^{26}$. If superoxide was indeed the reducing agent, the reaction of superoxide with CybB should be more complete at higher $\mathrm{pH}$ values. To test this, $\mathrm{KO}_{2}$ dissolved in anhydrous DMSO stabilized with 18-crown-6 ether was used to produce a stable superoxide stock solution. From this solution, a small amount was added to an aqueous solution containing detergent solubilized CybB $(1 \mu \mathrm{M})$ and the reduction of CybB was followed spectrophotometrically (mixing time $\sim 2 \mathrm{~s}$ ) at $428 \mathrm{~nm}$ at different $\mathrm{pH}$ values (Fig. $2 \mathrm{~A}$ ). Both the rate and the level of reduction increased with increasing $\mathrm{pH}$ correlating with a prolonged presence of superoxide at higher $\mathrm{pH}$ values (Fig. $2 \mathrm{~A}, \mathrm{~B}$ ). Essentially full reduction was observed at $\mathrm{pH}$ values $\geq 10$. If the reaction at $\mathrm{pH} 10$ was supplemented with SOD $\left(8.8 \mu \mathrm{g} \mathrm{ml}^{-1}\right)$, reduction of CybB was suppressed, indicating the superoxide specificity of the system. A further system that has been described to produce superoxide is NADH mixed with the electron mediator phenazine methosulfate $(\mathrm{PMS})^{21}$. Indeed, when this mixture was incubated with detergent solubilized CybB, rapid reduction of the hemes was observed and the reaction was completely suppressed in the presence of SOD (Fig. 2C). 
A

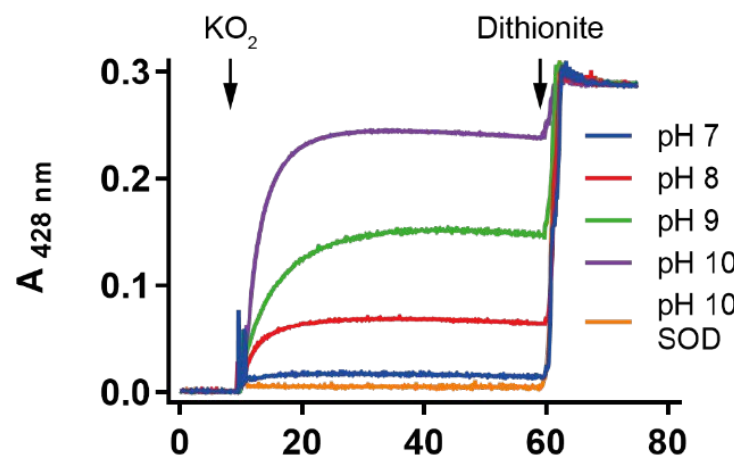

Time (s)
B

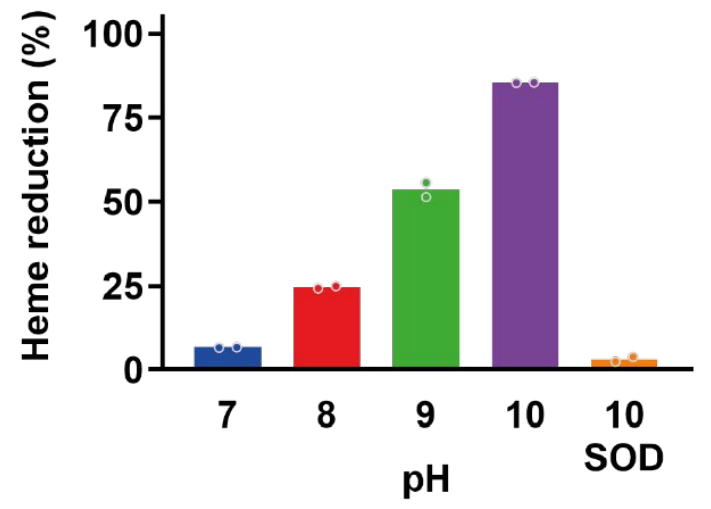

- $1 \mathrm{mM} \mathrm{NADH}$

- $0.1 \mathrm{mM} \mathrm{NADH}$

- $1 \mathrm{mM}$ NADH / $10 \mu \mathrm{M}$ PMS

- $0.1 \mathrm{mM} \mathrm{NADH} / 10 \mu \mathrm{M}$ PMS

- $1 \mathrm{mM}$ NADH / $2 \mu \mathrm{M}$ PMS

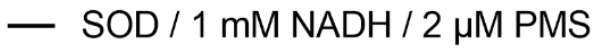

Figure 2: Superoxide CybB reduction. A) Kinetic traces monitoring CybB reduction at $428 \mathrm{~nm}$ upon addition of $5 \mu M$ superoxide from $\mathrm{KO}_{2}$ solution at different $\mathrm{pH}$ values. Full reduction of $\mathrm{Cy} b \mathrm{~B}$ was achieved by addition of sodium dithionite. $\left.B\right)$ percentage of the relative level of reduction after $20 \mathrm{~s}$ incubation with $\mathrm{KO}_{2}$ (Data from Fig. 2A). C) Kinetic traces monitoring $C y b B$ reduction in presence of various amount of $\mathrm{NADH}$ and $\mathrm{PMS}$ at $\mathrm{pH}$. NADH and SOD $\left(8.8 \mu \mathrm{g} \mathrm{ml}^{-1}\right)$ for the controls were added from beginning, PMS was added after $2 \mathrm{~s}$.

\section{Reaction of $C y b B$ with reduced ubiquinol}

To investigate the reverse reaction, we mixed detergent solubilized CybB (1 $\mu \mathrm{M})$ with ubiquinol $\mathrm{QH}_{2}$, prereduced with either $\mathrm{NaBH}_{4}$ or in situ by $1 \mathrm{mM}$ DTT 1 min prior to addition of the enzyme. CybB reduction by DTT alone is very slow and can be ignored (Fig. 3A). As depicted in Figure 3A, rapid reduction is observed if solubilized CybB is mixed with $1 \mathrm{mM}$ DTT and $10 \mu \mathrm{M}$ ubiquinone $\mathrm{Q}_{2}$ (purple trace). If $1 \mu \mathrm{M} \mathrm{Q}_{2}$ is used, the rate is slightly lower, but the same level of reduction is reached. At $0.1 \mu \mathrm{M} \mathrm{Q}_{2}$, the reaction was slowed down $>10$ times, and the maximum reduction was not reached within the measuring period. Next, we varied 
the $\mathrm{pH}$ of the reaction mixture, keeping, a more water-soluble analogue of $\mathrm{Q}_{2}$, DTT / $\mathrm{Q}_{1}$, constant at $1 \mathrm{mM} / 10 \mu \mathrm{M}$. As depicted in Fig. 3B, reduction levels of CybB were higher at alkaline $\mathrm{pH}$ values, while essentially no heme reduction was observed at $\mathrm{pH} 6$ or below. We had previously observed that upon the reaction with quinol, the enzyme consumes oxygen and produces superoxide, as detected with WST-1 ${ }^{17}$ (see also Fig. 6E). Consequently, under aerobic conditions, the enzyme is turning over, and the observed reduction level reflects steady-state catalysis. Therefore, the experiment was repeated under anaerobic conditions and while a similar trend was found, reduction levels were increased at all $\mathrm{pH}$ values compared to aerobic conditions (Fig. 3B). The difference between aerobic and anaerobic reduction was most pronounced at $\mathrm{pH}$ values $<7.5$. A plausible explanation for this behaviour is that the level of reduction correlates with the midpoint potential of the two reactants, the $b$ hemes of $\mathrm{CybB}$ and ubiquinone. The latter is strongly $\mathrm{pH}$ dependent ( $-60 \mathrm{mV}$ per $\mathrm{pH}$ unit), as two protons participate in the reaction ${ }^{27}$. A higher $\mathrm{pH}$ value thus corresponds to a lower midpoint potential, thus increasing the reducing power, while the midpoint potentials of protein embedded hemes are expected to be less sensitive to $\mathrm{pH}$ changes ${ }^{28}$.

A

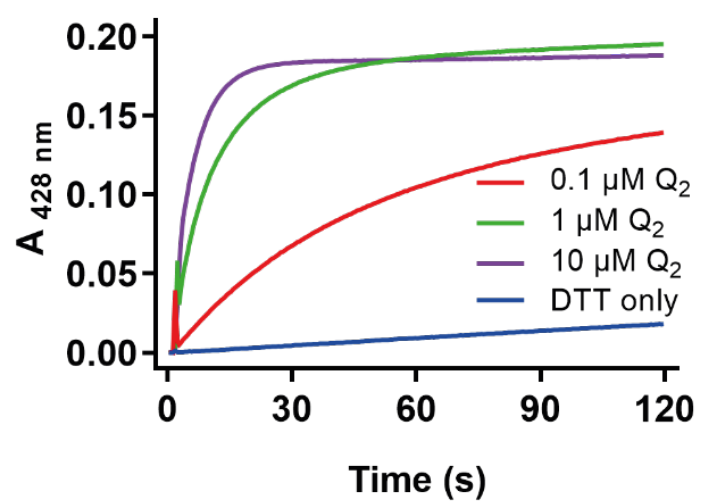

B

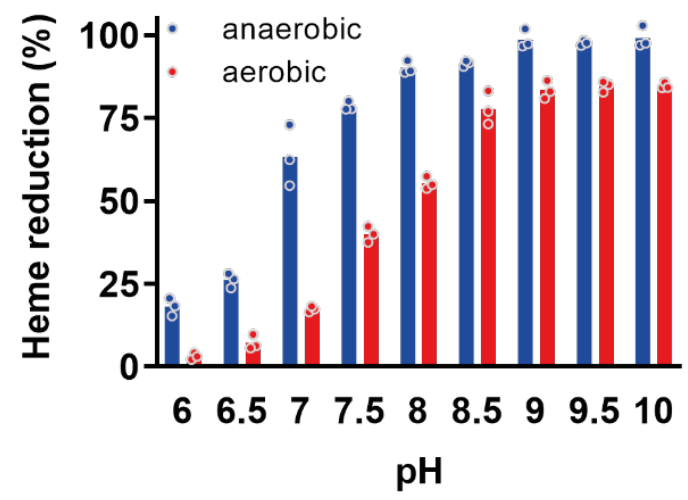

Figure 3: Quinol CybB reduction A) Kinetic traces monitoring CybB reduction at $428 \mathrm{~nm}$ upon addition of DTT alone or premixed with different amounts of ubiquinone $Q_{2}$ at $p H$ 8. B) percentage of heme reduction measured at $561 \mathrm{~nm}$ after $20 \mathrm{~s}$ incubation of CybB with $1 \mathrm{mM}$ DTT / $10 \mu M Q_{1}$ at different $\mathrm{pH}$ values under aerobic and anaerobic conditions.

\section{Redox titration of $C y b B$}

In $1986^{29}$, the midpoint potential of the two hemes was determined with the purified enzyme and a single value of $\sim 20 \mathrm{mV}$ was obtained at $\mathrm{pH}$ 7.4. However, it is unlikely that both hemes 
have an identical mid-point potential. If the forward reaction was favourable, a lower midpoint potential is expected for the periplasmic heme 2 compared to the cytoplasmic heme 1 to support electron transfer from superoxide via heme 2 and heme 1 to ubiquinone. We therefore performed a redox titration with dithionite under anaerobic conditions in the presence of a set of electron mediators and followed the potential with a voltmeter and the reduction state spectrophotometrically at $561 \mathrm{~nm}$. Several titrations were done in the absence and presence of ubiquinone $Q_{1}$, and the obtained traces, depicted in Figure $4 A$, were fitted for two hemes. The two waves have similar amplitudes, each of them reflecting $50 \%$ of the relative reduction signal as expected for two hemes with similar absorption properties. In the absence of ubiquinone, midpoint potentials of $-23 \pm 12 \mathrm{mV}$ and $+48.5 \pm 12.5 \mathrm{mV} \mathrm{NHE}$ were determined, close to the reported values, and we assigned them to heme 2 and heme 1 , respectively (Figure $1 B$ ). If ubiquinone $Q_{1}$ is present, the two potentials are shifted, one from $-23 \mathrm{mV}$ to $-8 \mathrm{mV}$ and the other from 48.5 to $100 \mathrm{mV}$. Importantly, the difference in midpoint potentials is increased $(108 \mathrm{mV})$, suggesting that binding of ubiquinone thermodynamically facilitates electron transfer from heme 2 to heme 1 (Fig. 4B). In addition, the increase of the potential at heme 2 facilitates reduction by superoxide, while the potential at heme $1(100 \mathrm{mV})$ is still fully compatible with quinone reduction. From these measurements, it can be speculated that binding of quinone to the oxidized enzyme could influence the reaction of the enzyme with superoxide. We therefore incubated CybB either with stoichiometric amounts or a 10-fold excess of quinone, before superoxide production was initiated by the addition of xanthine oxidase. As depicted in Figure $4 \mathrm{C}$, the observed reaction rate of the preincubated enzyme with superoxide increased dramatically. The reduction kinetics in presence of ubiquinone was biphasic, with a fast first phase and a slower second phase of reduction, which likely can be contributed to the reduction of CybB by ubiquinol (formed by either the adventitious reaction of superoxide with quinone or by CybB itself). To test this hypothesis, ubiquinol bos oxidase was added to keep the ubiquinone pool oxidised and prevent reduction by ubiquinol. The addition of $\mathrm{bo}_{3}$ oxidase resulted in a monophasic reduction of CybB with the first phase only, indicating that reduction occurred via superoxide only. 
A

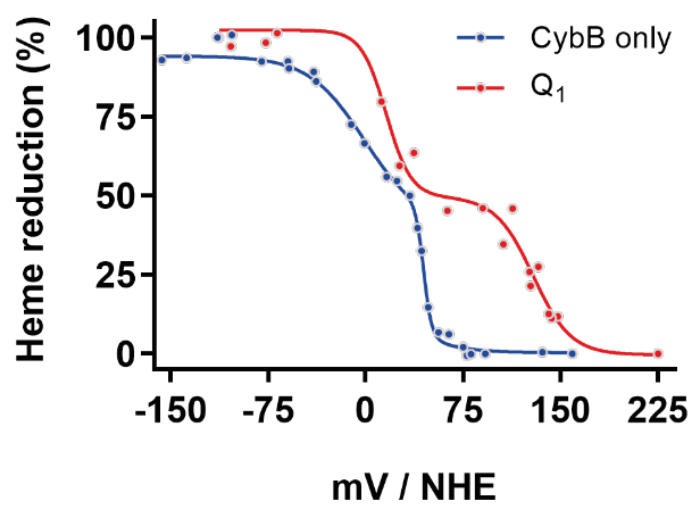

C

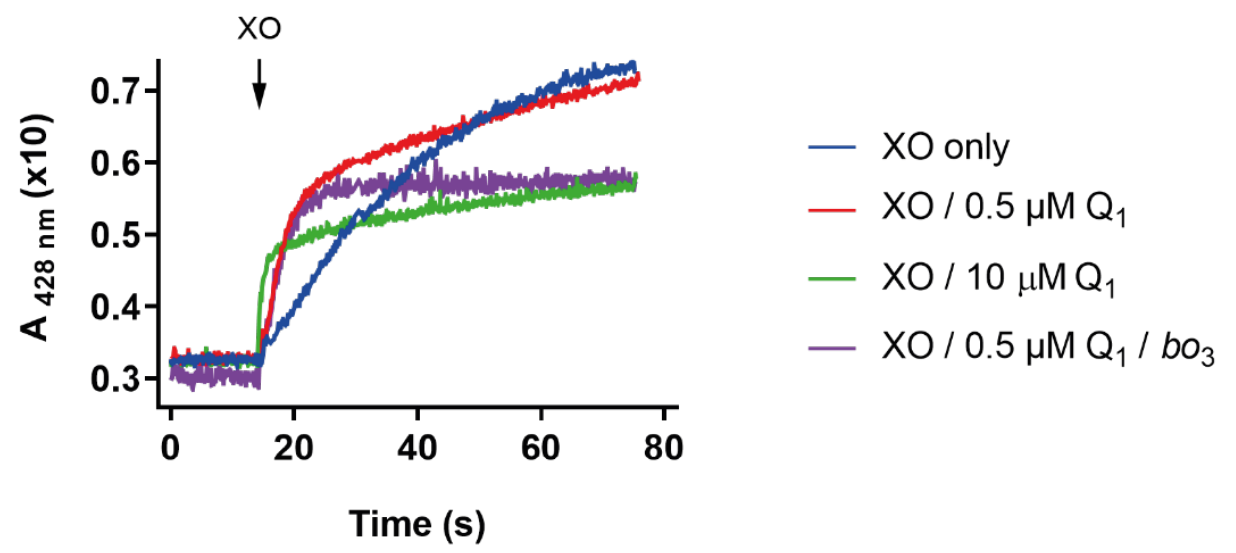

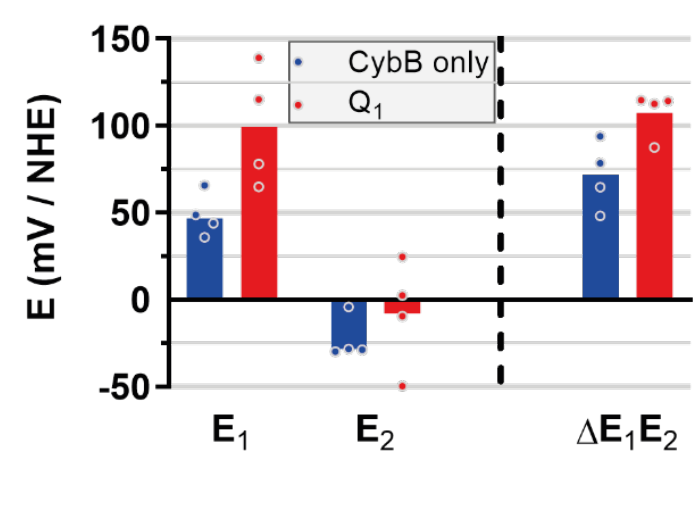

$E_{1} \quad E_{2} \quad \Delta E_{1} E_{2}$

B

(1)

Figure 4: Heme redox potentials. A) Midpoint redox potential titrations curves of $10 \mu M$ CybB in the absence (black) and in the presence (red) of $20 \mu M Q_{1}$. Redox potentials were performed anaerobically in $100 \mathrm{mM}$ sodium phosphate $\mathrm{pH} 7,0.1 \mathrm{mM}$ DTPA, $0.1 \mathrm{mM} \mathrm{HPX}$ and $0.05 \%$ DDM and redox potentials are expressed in $\mathrm{mV}$ against NHE. B) Energy diagram of potentials calculated from fits of Fig. $4 A$. Shown are the values for the low $\left(E_{2}\right)$ and high potential heme $\left(E_{1}\right)$ and their energy difference $\left(\Delta E_{1} E_{2}\right)$ in the presence and absence of quinone. C) Kinetic traces showing reduction of $0.5 \mu M$ of CybB by superoxide anion produced by xanthine oxidase in the absence and in presence of an equimolar (0.5 $\mu \mathrm{M})$ or an excess amount (10 $\mu \mathrm{M})$ of ubiquinone $Q_{1}$ and $60 \mathrm{nM}$ of $b_{3}$ oxidase. Measurement was done in $100 \mathrm{mM}$ sodium phosphate $\mathrm{pH} 8,0.1 \mathrm{mM}$ DTPA, 0.1 $m M H P X$ and $0.05 \%$ DDM.

\section{Both ubiquinone and menaquinone are substrates of $C y b B$}

In contrast to the reverse reaction, the forward reaction is more complicated to monitor due to the short half-life of superoxide and its hard-to-control production. We have therefore simplified the system and pre-reduced CybB under anaerobic conditions with stoichiometric amounts of dithionite, until $~ 90-95 \%$ of the enzyme is reduced to avoid presence of unreacted dithionite. 
A

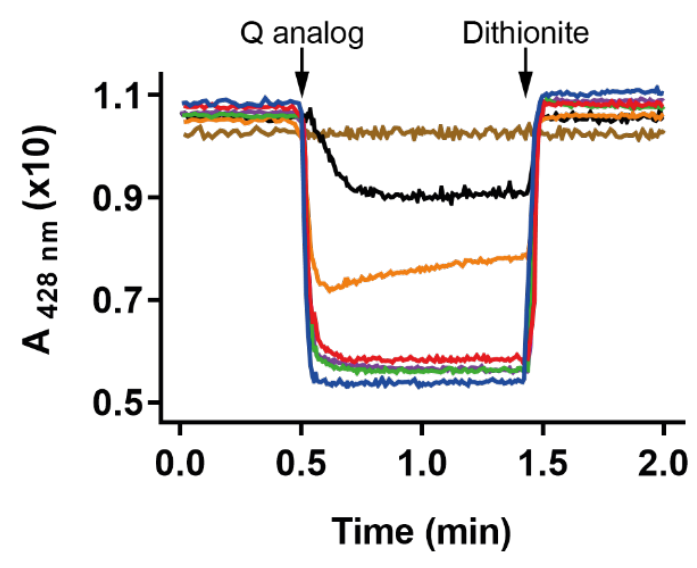

C

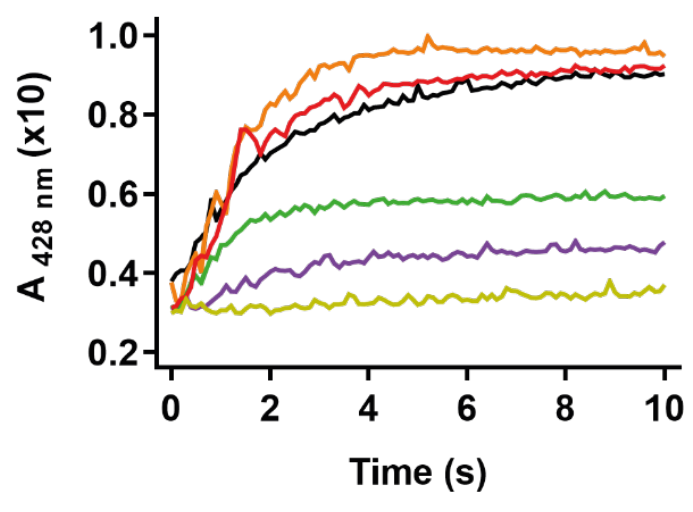

B

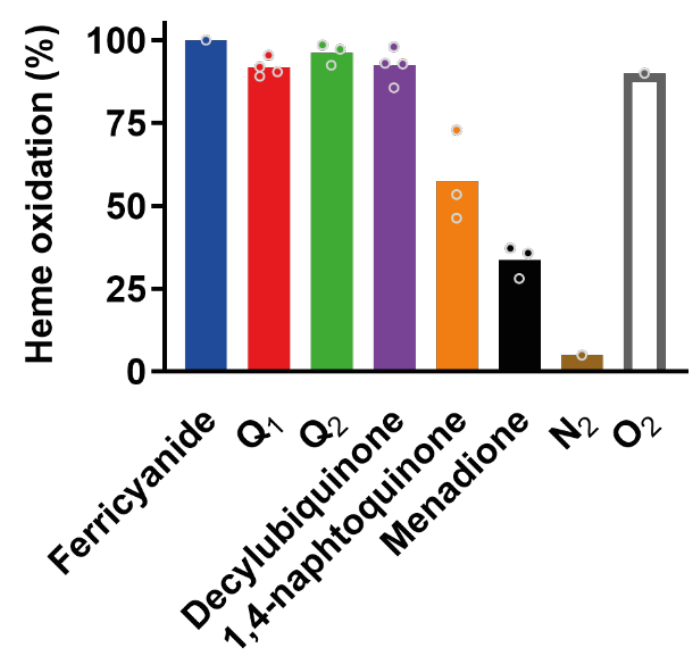

D

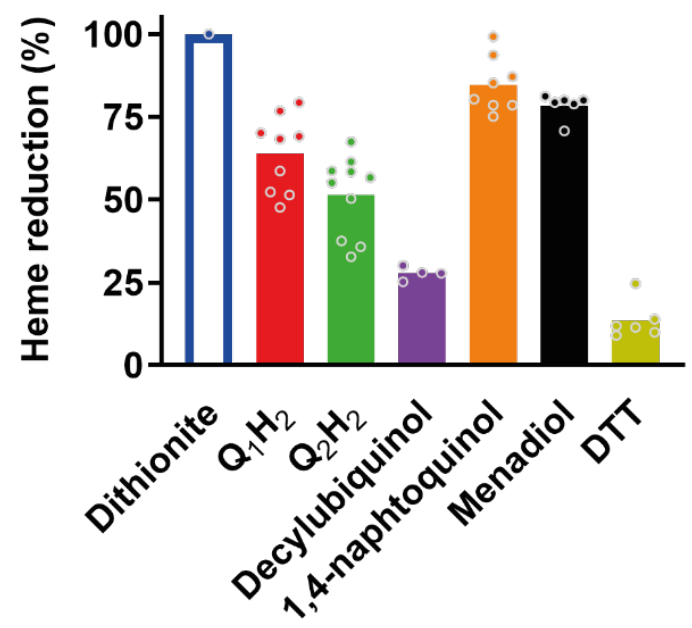

Figure 5: Ubiquinone and menaquinone as substrates for CybB. A) Kinetic traces of CybB oxidation as observed at $428 \mathrm{~nm}$. CybB was reduced, under anaerobic conditions, with small amounts of dithionite (6.6 $\mu M)$ until $>90$ $\%$ reduction was obtained, after which $10 \mu \mathrm{M}$ of different quinones was added. After the reaction was complete, $C y b B$ was fully reduced by addition of $3.3 \mathrm{mM}$ dithionite. B) Summary of the percentage of reduced heme oxidation, including the data from addition of air-saturated water (not included in Fig. 1A). C) Kinetic traces of $C y b B$ reduction, under aerobic conditions, as observed at $428 \mathrm{~nm}$. Different quinols were added at $10 \mu \mathrm{M}$ concentration. D) Summary of the results obtained from Fig. 5C. (Note that the reference sample (Dithionite) has no trace in $5 C)$. The bars in figures $5 B$ and $5 D$ have the same color as the respective oxidized (5A) or reduced (5C) quinone traces.

Addition of ubiquinone $10 \mu \mathrm{M} \mathrm{Q}_{1}$ showed a rapid oxidation of $\mathrm{CybB}$, indicating that ubiquinone is a substrate (Fig. 5A, red trace). A similar behavior was found with other shortchain ubiquinones such as $Q_{2}$ and decylubiquinone. Next, we tested the two menaquinone analogues menadione and 1,4-naphtoquinone. Reaction with 1,4-naphtoquinone proceeded also quickly, but enzyme oxidation was less complete, while the reaction with menadione was 
$>10$ slower and less enzyme was oxidized. A summary of the different experiments is found in Figure $5 B$, showing that reaction with ubiquinones reached almost complete CybB oxidation, in reference to the oxidation by ferricyanide. Addition of air saturated water also led to oxidation of $\mathrm{CybB}$, indicating that the reduced enzyme can deliver electrons to oxygen in the absence of quinones.

Next, we compared the ability of the reduced form of these different quinones to reduce CybB under aerobic conditions, i.e., the reverse reaction. When using ubiquinol $\mathrm{Q}_{1} \mathrm{H}_{2}$ at $\mathrm{pH} 8, \sim 60$ $\%$ of reduction of CybB was observed as discussed above (Fig. 5C). Again, the observed kinetics were very similar to $\mathrm{Q}_{2} \mathrm{H}_{2}$ and decylubiquinol, but both quinols showed a lower level of reduction. In contrast to the forward reaction, incubation with the reduced menaquinol analogues led to almost complete reduction of the enzyme (Fig. 5 C, D). Hence, the observed data correlate well with our hypothesis that the lower midpoint potential of menaquinones (compared to ubiquinones), leading to a more complete reduction of CybB.

Measurement of steady state activity of the forward reaction and determination of quinone affinity

Having the two most likely substrates identified, we aimed to develop a turnover assay that reports the actual protein function, converting superoxide to reduced quinol. However, it is not trivial to directly measure quinol formation (see discussion). We therefore adapted a superoxide dismutase assay using WST-1, a water-soluble indicator for superoxide, which after reaction with superoxide is converted into a stable formazan that strongly absorbs at $438 \mathrm{~nm}^{17}$. As a constant superoxide producing system, xanthine oxidase and hypoxanthine was employed, which in phosphate buffer has been reported to produce $\sim 50 \%$ superoxide and $50 \%$ hydrogen peroxide ${ }^{30,31}$. Although neither CybB nor WST-1 has been shown to react with hydrogen peroxide, catalase is included in the assay buffer to avoid accumulation of hydrogen peroxide. Consequently, the linear increase observed at $438 \mathrm{~nm}$ represents the continuous conversion of WST-1 by xanthine oxidase produced superoxide into the stable formazan (Fig. 6B, blue trace). Winterbourn and others have previously used this assay to estimate SOD content of tissue extracts, as any present SOD competes with WST-1 for superoxide leading to a decreased formazan formation ${ }^{22}$. We adapted this idea and added the required components for CybB to compete with WST-1 for superoxide. If CybB (50 nM) and $\mathrm{Q}_{2}(10 \mu \mathrm{M})$ were present (orange trace on Fig. 6), the initial signal was a bit lower, but 
quickly regained the initial slope. We interpreted this result as competition between CybB and WST-1 for superoxide that stops after enough ubiquinone has been converted to ubiquinol. We therefore added purified $\mathrm{E}$. $\mathrm{Coli}_{\mathrm{bo}}$ quinol oxidase to the mixture to regenerate the quinone pool, which led to a continuous suppression of formazan production (red trace on Fig. 6). Importantly, if $b_{3}$ and $Q_{2}$ were present in the absence of $C y b B$, no suppression was observed (green trace on Fig. 6). This indicates that the spontaneous reaction of superoxide with ubiquinone to semiubiquinone - accompanied by the disproportionation to quinone and quinol (that can be consumed by the $b_{3}$ oxidase) - is not sufficient to explain the observed suppression. The overall scheme of the assay with all components is depicted in Figure 6A.

We used this approach to estimate both the second order rate constant of the reaction and the apparent $K_{m}$ for ubiquinone. First, in the presence of all components including $10 \mu \mathrm{M} \mathrm{Q} \mathrm{Q}_{2}$ and $\mathrm{bo}_{3}$ oxidase, different amounts of SOD and $\mathrm{CybB}$ were added and the decrease in formazan formation was followed. As depicted in Figure 6C, about 10-times more CybB was required to obtain similar quenching values. Taking the previously determined second order rate constant of $2 \times 10^{9} \mathrm{M}^{-1} \mathrm{~s}^{-1}$ for SOD, a rate constant in the range of 1 to $3 \times 10^{8} \mathrm{M}^{-1} \mathrm{~s}^{-1}$ can be estimated for CybB (see table 1). To measure the apparent $K_{m}$ of the reaction for ubiquinone, all components except ubiquinone were mixed, and a steady formazan formation was monitored for $30 \mathrm{~s}$, before different amount of $\mathrm{Q}_{2}$ were added from stock solutions, leading to decreased levels of formazan formation (Figure 6D). Using Michaelis-Menten kinetics, an apparent $K_{m}$ of $390 \pm 40 \mathrm{nM}$ was obtained. We determined very similar $K_{m}$ values by either subsequent addition of increasing amounts of quinone as well as with different quinone concentrations measured in parallel in a 96-well plate.

Finally, we also determined an apparent $K_{m}$ for the reverse reaction. To this end, superoxide formation by CybB was monitored following formazan formation from WST-1 (Fig. 6E and supplementary Fig. S3) after addition of $\mathrm{NaBH}_{4}$-prereduced ubiquinol. As depicted in Figure $6 \mathrm{~F}$, if initial rates were plotted against quinol concentrations, an apparent $K_{m}$ of $\sim 10 \mu \mathrm{M}$ was obtained, thus being 25 higher than for the forward reaction (see table 1). 
A

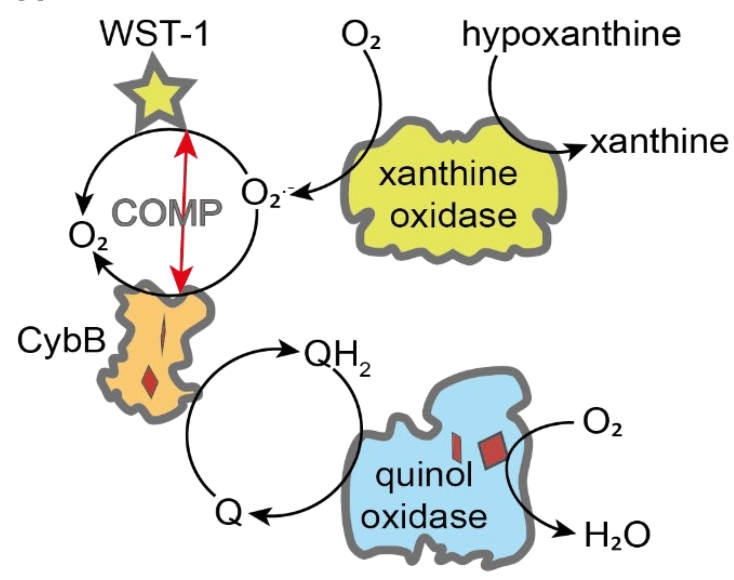

C

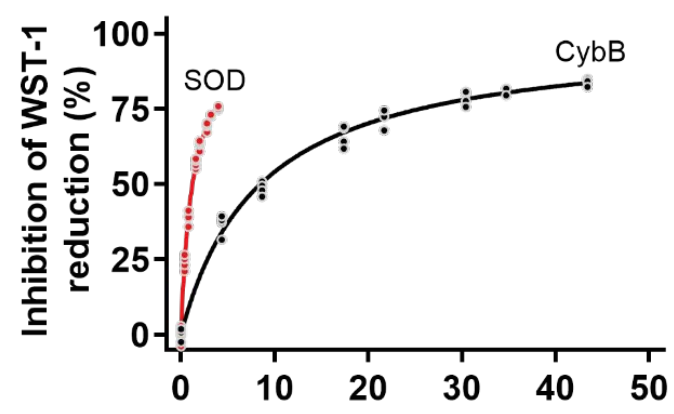

Enzyme concentration (nM)

\section{E}

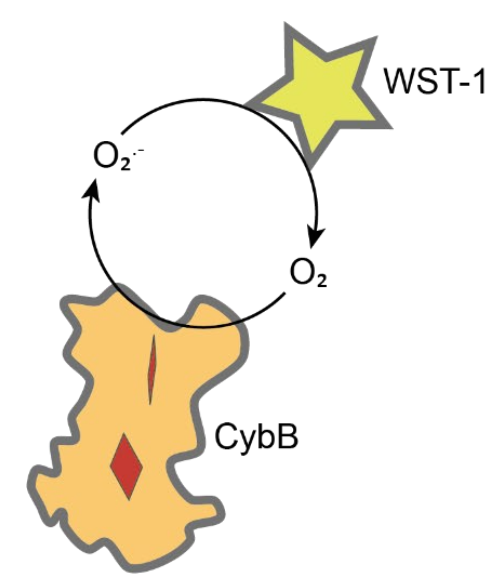

B

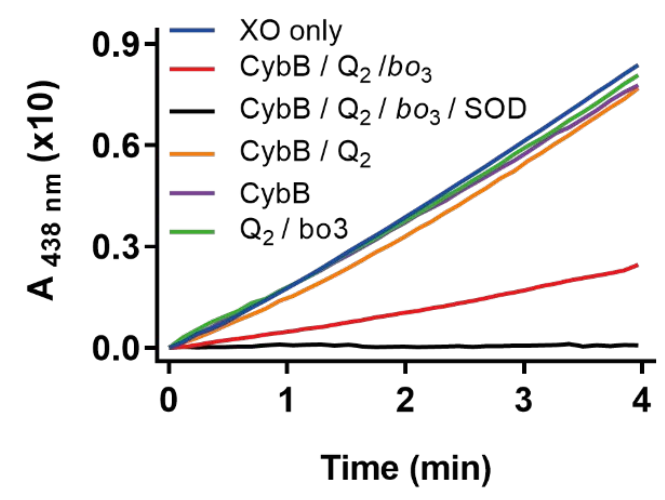

D

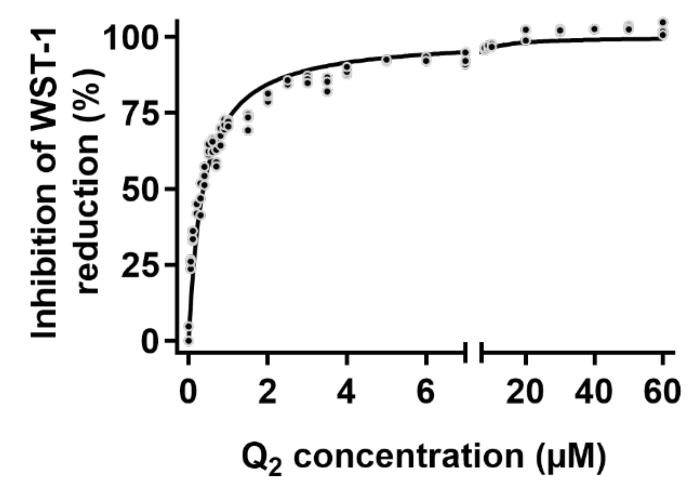

$\mathbf{F}$

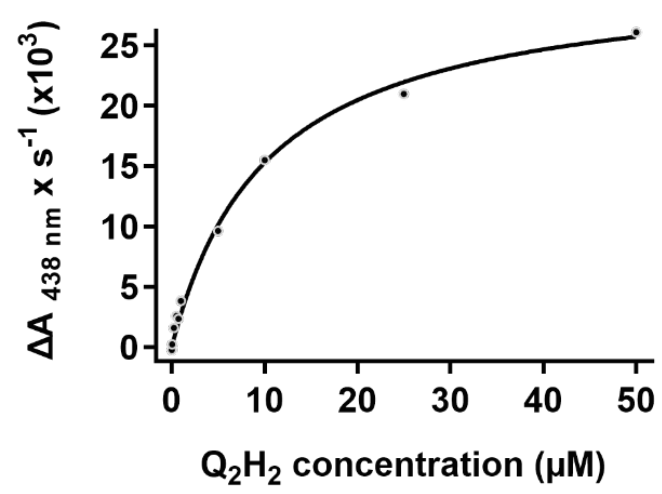

Figure 6: Superoxide assay and apparent $K_{m}$ determination. A) Cartoon depicting the coupled assay to follow competition (COMP) between CybB and WST-1 for superoxide. B) Kinetic traces monitoring formazan formation at $438 \mathrm{~nm}$. The different conditions are indicated in the legend. C) Titration of different amounts of purified CybB and purified SOD under otherwise identical experimental conditions ( $0.01 \mathrm{U}$ xanthine oxidase, $10 \mu \mathrm{M} \mathrm{Q} \mathrm{Q}_{2}, 60 \mathrm{nM}$ bo $\mathrm{o}_{3}$ oxidase). Relative decrease in formazan rate formation (inhibition of WST-1 reduction \%) upon addition of either SOD (red trace) or CybB (black trace). D) Determination of the apparent $K_{m}$ of ubiquinone $Q_{2}$ in the forward reaction. Relative decrease in formazan rate formation upon addition of different amounts of $Q_{2}$. E) Cartoon depicting the assay scheme to determine reverse activity, i.e., formation of superoxide by quinol. F) Determination of apparent $K_{m}$ of ubiquinol $Q_{2} H_{2}$ in the reverse reaction. Initial slopes of formazan formation $(\triangle A$ ${ }_{438 \mathrm{~nm}} \times \mathrm{S}^{-1}\left(\times 10^{3}\right)$ ) upon addition of different amounts of prereduced ubiquinol $\mathrm{Q}_{2} \mathrm{H}_{2}$ (as depicted in Supplementary Fig. S3). 


\section{Discussion}

\section{Architecture of a superoxide: quinone oxidoreductase}

The enzyme described here, $C y b B$ or cytochrome $b_{561}$ from $E$. coli was proposed to act as a membrane bound superoxide:ubiquinone oxidoreductase ${ }^{17}$. It is only the third enzyme described, after SOD and SOR, to specifically react with superoxide. However, in contrast to the two other enzymes that contain a catalytic dimetal center, CybB is a hemoprotein containing two $b$ hemes. Superoxide produced in biological systems is either the product of an enzymatic or adventitious reaction of an electron with molecular oxygen. It has a standard midpoint potential of $-160 \mathrm{mV}$, which however is expected to be much higher under biological conditions as the $\mathrm{O}_{2} / \mathrm{O}_{2} \cdot-$ ratio is expected to be very large in the cell (e.g., a ratio of $\mathrm{O}_{2} / \mathrm{O}_{2}{ }^{--}$ $=1,000$ the potential increases to $\sim+42 \mathrm{mV}$ ). Superoxide spontaneously disproportionates into oxygen and hydrogen peroxide, which are more stable products. In principle, superoxide can act as a mild reductant, however, it is better known as an oxidant oxidizing lipids or enzyme metal centers ${ }^{6}$. Superoxide reacts with many organic molecules in solution, including ubiquinone. The product, ubisemiquinone, has been shown to disproportionate into ubiquinone and ubiquinol ${ }^{32}$. However, this spontaneous reaction is not expected to occur in biological systems, as superoxide is considered membrane impermeable due its negative charge, while ubiquinones are tightly embedded in the membrane. Superoxide production can also be envisioned to happen at the membrane surface as oxygen preferentially partitions into the membrane, and quinone binding sites of respiratory enzymes have been described as point of superoxide formation ${ }^{3,33-35}$. In a similar scenario, water-soluble ascorbate and membrane-bound ubiquinone are unable to react in cells, even though they have been shown to rapidly react in solution ${ }^{36}$. The proposed design of a membrane embedded superoxide:quinone oxidoreductase therefore makes sense if electron transfer between the two otherwise separated substrates should happen. The orientation of CybB in the membrane predicts that reduction occurs from the periplasm via the hemes to the quinone binding site located on the cytoplasmic side. The idea of a quinone binding site is consistent with the glycerol found in the crystal structure and docking studies ${ }^{17}$. The binding of superoxide from the periplasmic side is supported by an easily accessible heme from the protein surface surrounded by positively charged amino acids. Such a positive patch would attract, and guide, negatively charged superoxide anion radical $\mathrm{O}_{2} \cdot$, creating an entry path for superoxide. 
Similar positive patches have also been found in the human $\mathrm{Cu}, \mathrm{Zn}-\mathrm{SOD}^{37}$, Alvinella pompejana $\mathrm{Cu}, \mathrm{Zn}-\mathrm{SOD}^{38}$ or the superoxide reductase (SOR) of Desulfoarculus baarsii in complex with $\mathrm{Fe}(\mathrm{CN})_{6}{ }^{39,40}$. However, the strongest indication for a specific intercatio006E of superoxide with CybB is the recent structure of the transmembrane domain of the NADPH oxidase (Nox5) from Cylindrospermum stagnale 7,41 . The protein consists of a transmembrane domain formed by six helices, with an overall similar fold as $\mathrm{CybB}$, harboring two $b$ hemes in close distance. The protein protrudes into the cytoplasm with an NADPH binding domain containing FAD as cofactor. This topology allows the enzyme to use cellular NADPH to produce superoxide and hydrogen peroxide on the extracellular side to act as chemical weapon in cellular warfare to combat bacterial infections. Notably, the two proteins, Nox5 and CybB, show a very similar electrostatic environment around the periplasmic heme, which is surrounded by positively charged amino acids creating an easy access route for negatively charged species in the periplasmic compartment (see Fig. 7). In particular, it has been proposed that Arg256 present in this cavity could electrostatically promote the catalytic production of superoxide ${ }^{7}$. The overall reaction of Nox5, oxidation of NADPH and reduction of oxygen to superoxide is thus very similar to the reverse reaction of $\mathrm{CybB}$, ubiquinol oxidation and superoxide formation, and the structural arrangement of the two enzymes, especially with respect to the exposed heme and the positive patch, is also strikingly similar.

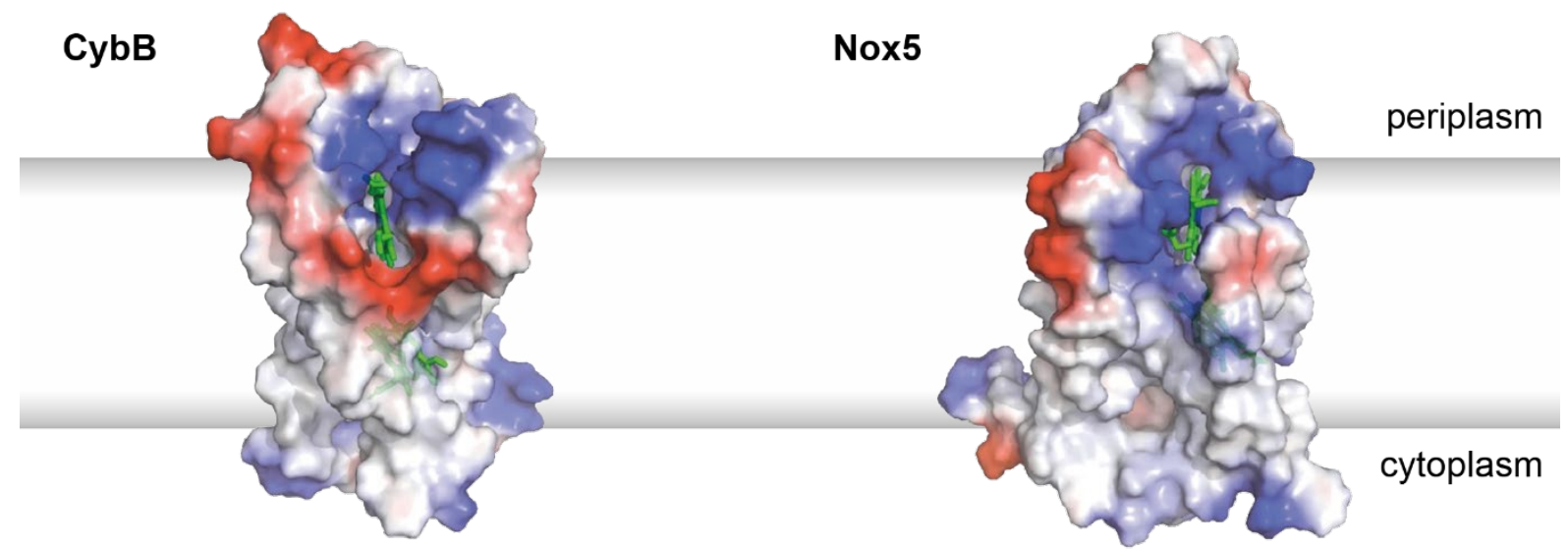

Figure 7: Comparison of the electrostatic potential map. The charges distribution around the exposed edge of heme group (green) of CybB ( $p d b 50 C 0$ ) and Nox5 ( $p d b 500 T$ ) show a similar positive surface potential (blue) that is presumed to funnel the superoxide anion to the heme edge. Images were produced in Pymol. 


\section{Reaction of CybB with its substrates}

A short description of enzyme purification is included in the results section for a reason. Even though the purified CybB was very pure on SDS-PAGE gel immediately after purification via $\mathrm{Ni}-I D A$ resin and the reduced dithionite spectra appeared identical to those after gel filtration, functional characterization revealed different kinetics and levels of heme reduction in different enzyme preparations when reduced with DTT / Q1 or superoxide. We therefore performed the described experiments by varying detergent and IDA elution methods, until we found conditions that yielded protein with reproducible properties. Possible reasons for the inconsistent behaviour in the other preparations are different levels of delipidation and protein aggregation. It can be expected that a small protein with such a distorted structure relies on interactions with its annular layer of lipids, and this requires further investigation in the future.

With the optimized enzyme preparation in our hands, the experiments presented here provide strong evidence that superoxide and both ubiquinone and menaquinone are natural substrates of CybB. This quinone substrate diversity is not surprising, as all terminal $E$. coli oxidases $\left(b_{3}, b d l\right.$ and $\left.b d l l\right)$ have been shown to accept either $\mathrm{UQ}_{8}, \mathrm{DMQ}_{8}$ or $\mathrm{MQ}_{8}{ }^{42}$, as well as $E$. coli the nitrate reductase (NarGHI) during nitrate respiration ${ }^{43-45}$. We show that $\mathrm{CybB}$ is readily reduced by short chain ubiquinols in a strongly $\mathrm{pH}$ dependent manner. The standard midpoint potential of ubiquinone / ubiquinol is $\sim 70 \mathrm{mV}$ at $\mathrm{pH} 7^{46}$, but decreases by $-60 \mathrm{mV}$ per $\mathrm{pH}$ unit, rendering it a stronger reductant at higher $\mathrm{pH}$ values in accordance with our data. We also find that the observed steady state reduction is increased under anaerobic conditions, in agreement that in the absence of oxygen the enzyme cannot turnover leading to an accumulation of a reduced intermediate. We show that menaquinols, that have a lower midpoint potential $(\sim-100 \mathrm{mV})$, reduce the enzyme more completely than ubiquinol. The opposite picture is observed, if the prereduced enzyme is mixed with the oxidized form of the different quinone analogues under anaerobic conditions. While incubation with oxygen and ubiquinone led to a completely oxidation comparable to ferricyanide, incubation with the lower potential menaquinone led only to a partial oxidation of the enzyme, corroborating that the observed level is dependent on the redox potential of the substrate. In contrast to ubiquinone, the redox potential of the two heme groups are not expected to be heavily $\mathrm{pH}$ dependent, as no proton takes place in the redox reaction. 
The specific reaction of CybB with superoxide is more complex to analyse because of difficulties to produce sufficient concentrations due to the instability of superoxide. At $\mathrm{pH} 8$, the half-life of $10 \mu \mathrm{M}$ superoxide is approximately $1 \mathrm{~s}^{26}$. Here, we have used three wellestablished methods to produce superoxide in aqueous solutions, whilst superoxide specificity was tested by signal suppression after the addition of $\mathrm{SOD}^{22,47,48}$. First, superoxide was produced enzymatically by xanthine oxidase, which is routinely used to investigate SODs. However, it is nearly impossible to quantify the superoxide directly, as the percentage of superoxide produced per hypoxanthine molecule varies strongly and depends on the buffer conditions and on the enzyme preparation itself ${ }^{31}$. The activity of commercially available xanthine oxidase must thus be analysed for every purchased batch. Second, we used inorganic potassium dioxide mixed with a crown-ether that produces a stable superoxide solution in organic solvents, e.g., DMSO or acetonitrile and finally we used NADH and phenazine methosulfate (PMS), which produces superoxide in aqueous solution ${ }^{21}$. All three systems rapidly reduced $\mathrm{CybB}$ and, in all cases, addition of SOD suppressed CybB reduction. To exclude unspecific reaction of superoxide with other heme-containing enzymes, we incubated purified $b o_{3}$ oxidase with the xanthine oxidase / hypoxanthine and found essentially no heme $b$ reduction over the time course of CybB reduction (Supplementary Fig. S2). In the case of $\mathrm{KO}_{2}$, we analysed the $\mathrm{pH}$ dependency of the reaction and found that $\mathrm{CybB}$ can be fully reduced at higher $\mathrm{pH}$ values. Since the redox potential of the oxygen / superoxide couple is not proton dependent, we attribute this observation to the increased stability of the superoxide anion (and hence a higher substrate concentration). It is important to point out that all tested low redox potential substrates such as $\mathrm{NADH}(-340 \mathrm{mV})$, DTT $(-330 \mathrm{mV})$, glutathione $(-240 \mathrm{mV})$, lactate $(-160 \mathrm{mV})$ or hypoxanthine $(-400 \mathrm{mV})$ were unable to reduce the enzyme at $\mathrm{pH} 8$. Earlier reports that $\mathrm{NADH}$ and lactate reduce $\mathrm{CybB}$ in membrane vesicles are likely due to adventitious superoxide production during respiration by NADH and lactate dehydrogenases, respectively ${ }^{49}$. A notable exception is ascorbic acid $(+60 \mathrm{mV})$, described as natural substrate for many other cytochrome $b_{561}$ proteins, which reduced CybB of $E$. coli slowly and in a $\mathrm{pH}$ dependent fashion (no reduction below pH 7), likely due to its decreased potential at higher $\mathrm{pH}$ values. Interestingly, in cytochrome $b_{561}$ of secretory vesicles that regenerate internal ascorbate from semidehydroascorbate via transmembrane electron transfer, the reduction of the enzyme is not $\mathrm{pH}$ sensitive between $\mathrm{pH} 6$ and 8 , indicating that a different mode of action with ascorbate is utilised in this enzyme compared to $\mathrm{CybB}^{50}$. 


\section{Midpoint potential of CybB hemes}

We determined the midpoint potential of the two hemes by titrating dithionite to an anaerobic solution of CybB in the presence of electron mediators. While Murakami et $\mathrm{al}^{29}$ found a single potential at $+20 \mathrm{mV}$ in a similar experiment, we were able to fit the titration to two waves with $\sim 50 \%$ signal amplitude each, indicating that the two hemes have different potentials, i.e. $-23 \mathrm{mV}$ and $+48.5 \mathrm{mV}$. These values are close to the ones reported by Murakami and well within the range found for other diheme $b$ enzymes, i.e. heme $b$ in $E$. coli $b_{3}$ oxidase and nitrate reductase have been shown to be $+50 \mathrm{mV}^{51}$ and $+20 \mathrm{mV}^{52}$, respectively. For diheme proteins of the cytochrome $b_{561}$ family, typically slightly higher potentials have been found. In cytochrome $b_{561}$ from bovine adrenal chromaffin vesicles, the redox potentials of high and low potential hemes were determined to be +150 and $+60 \mathrm{mV}$, respectively ${ }^{53}$. A value of $+80 \mathrm{mV} \pm 30 \mathrm{mV}$ has been reported for the human duodenal cytochrome $b_{561}$ that catalyse transmembrane electron transfer from external ascorbate to intravascular $\mathrm{Fe}^{3+54}$. In plants, the reported midpoint redox potential cytochrome $b_{561}$ proteins range between +80 to $+190 \mathrm{mV}$ for the high potential heme and between -20 to +60 $\mathrm{mV}$ for the low potential heme, in accordance with the potentials of the proposed substrate couple dehydroascorbate / ascorbate $(+60 \mathrm{mV})^{55}$. In the case of CybB, the measured midpoint redox potentials of $-23 \mathrm{mV}$ and $+48.5 \mathrm{mV}$ are assigned to periplasmic side faced heme 2 and cytoplasmic faced heme 1, respectively (Figure $1 \mathrm{~A}$ ). Repetition of the potential measurements in the presence of $20 \mu \mathrm{M}$ ubiquinone $Q_{1}$ primarily shifted the midpoint potential of heme 1 to higher values, increasing the potential difference between the two hemes from $+64 \mathrm{mV}$ to $+108 \mathrm{mV}$, supporting fast intramolecular electron transfer ${ }^{56}$. We thus hypothesize that binding of the substrate quinone close to heme 1 increases its potential, facilitating electron transfer from heme 2 to heme 1 . We tested this hypothesis by comparing the reaction of CybB with superoxide in the presence and absence of quinone. In the presence of saturating quinone concentration the reaction was faster than the mixing time $\left(t_{1 / 2}<1 \mathrm{~s}\right)$. This effect is in accordance with the observation that binding of the semiquinone analogue inhibitor 'HQNO' decreased the potential of the low potential heme in succinate: menaquinone oxidoreductase from Bacillus subtilis ${ }^{57}$. Future experiments using EPR spectroscopy will help to measure and assign heme potentials reliably and pulse radiolysis studies will enable fast reaction kinetics of CybB with superoxide. 
Table 1: Summary of kinetics parameters as determined using WST-1 assay.

\begin{tabular}{cccc}
\hline Substrate & $V_{\max }\left(\mathbf{A}_{\left.438 \mathrm{~nm} . \mathrm{s}^{-1}\right)}\right.$ & $\boldsymbol{K}_{m}(\mu \mathrm{M})$ & Second order rate $\left(\mathrm{M}^{-1} \mathrm{~s}^{-1}\right)$ \\
\hline Ubiquinone $\mathrm{Q}_{2}$ & $37.49 \pm 0.68$ & $0.39 \pm 0.04$ & $\begin{array}{c}2.9 \pm 0.4 \times 10^{8} \\
\left(\mathrm{SOD}=2.0 \times 10^{92}\right)\end{array}$ \\
\hline Ubiquinol $\mathrm{Q}_{2} \mathrm{H}_{2}$ & $31.02 \pm 2.79$ & $10.29 \pm 2.40$ & nd \\
\hline
\end{tabular}

\section{Enzyme turnover measurements}

The strongest evidence for the proposed reaction was obtained in a coupled enzyme assay that shows that CybB actively competes with WST-1 for superoxide and that this competition is dependent on the CybB and quinone concentrations, as well as on quinone regeneration by $b_{3}$ oxidase. This assay has been used and validated to detect SOD activity in tissues and purified fractions. Here, we confirm that only in the presence of all components, suppression of formazan formation was observed. If all components but CybB was present, WST-1 reduction by xanthine oxidase was unaffected. This is noteworthy, as it has been described that superoxide reacts with ubiquinone to form semiquinone, which can disproportion to ubiquinol and oxygen. Alternatively, semiquinone can react with oxygen to form superoxide again. However, if this reaction occured at a significant rate, formazan formation would already be decreased in the presence of quinone and $b_{3}$ oxidase (without CybB), which was not the case. Whether the added detergent affected this reaction, or the disproportion reaction was slow compared to the reaction of semiquinone with oxygen is unclear. To eliminate potential pitfalls, we have used the exact same conditions, including the presence of quinone and $\mathrm{bo}_{3}$ oxidase, and performed the experiment with purified SOD. We were surprised to find that only $10 \mathrm{x}$ more CybB than SOD was required to suppress WST-1 formation to a similar extent. This suggests that the second order rate constant of CybB is about 10 times lower than the one of SOD, yielding a still impressive value of $\sim 2.9 \times 10^{8} \mathrm{M}^{-1} \mathrm{~s}^{-}$ ${ }^{1}$ in the presence of $10 \mu \mathrm{M}$ ubiquinone $\mathrm{Q}_{2}$ (see table 1 ). Such a rate makes CybB a true competitor for cellular superoxide even in the presence of SOD (see below).

This same assay was used to titrate quinone as a substrate, rendering a $K_{m}$ of $\sim 390 \mathrm{nM}$, which is lower than what has been found for other quinone reducing enzymes in bacteria (typically in the micromolar range) ${ }^{58-62}$. Our data was analysed using Michaelis-Menten, which might be an oversimplification, as we do not know the "excess" of substrate superoxide over 
enzyme, which is further influenced by the spontaneous disproportion of superoxide. Obviously, such high turnover rates require a fast association of $\mathrm{CybB}$ with superoxide and a fast reduction of ubiquinone, and the rates of these have not been measured yet. In future experiments, pulse radiolysis will be used as an efficient mean to rapidly produce defined amounts of superoxide and investigate pre-steady-state kinetics on the enzyme as well as stopped-flow measurements to follow the oxidation of the enzyme by quinone. However, it might well be that superoxide binding to the quinone-bound enzyme is rate limiting, and subsequent electron transfer might be very fast, and that CybB intermediates with reduced hemes are thus not accumulating. The prerequisite of the quinone binding to CybB before the electron transfer from superoxide takes place might reflect a strategy to suppress reactive intermediates in the membrane. Mechanistically, it is important to remember that two molecules of superoxide must bind to fully reduce quinone to quinol and that protons must be taken up before reduced quinol can be released to the membrane and be used as a substrate for e.g., respiration. Future mutagenesis studies should allow us to identify the residues that are critical for these processes.

\section{Concluding remarks}

The presented data strongly support our initial hypothesis, which was based on the crystal structure and a set of functional experiments ${ }^{17}$ that CybB of $E$. coli can work as a superoxide oxidase in vitro. While fast pre-steady state measurements like those performed for SOD and SOR are still outstanding, we find the present set of data corroborates our initials findings to be convincing. The measured redox potentials of the two hemes are lower than those of other cytochrome $b_{561}$ proteins, suggesting a different substrate than ascorbate. In addition to the proposed forward reaction, also the reverse reaction (formation of superoxide from ubiquinol) is readily observed, and the similarity of the structural arrangement to the NADPH: superoxide oxidase is intriguing. The apparent $K_{\mathrm{m}}$ for quinone is 25 times lower in the forward direction, and for quinol in the reverse direction, making the forward reaction kinetically favourable.

To date, the physiological role of CybB is unknown, as simple growth experiments have not yielded a phenotype in a knockout strain. It seems unlikely, however that the forward reaction takes place under aerobic conditions, when the oxygen / superoxide ratio is high $\left(>10^{6}\right.$ in a laboratory culture). Under such conditions, the reverse reaction is more likely to occur. In this 
context, it is noteworthy that periplasmic superoxide formation has been described for $E$. coli $^{12}$, and the reaction of menasemiquinone with oxygen has been suggested as a pathway for this. Based on our results with menaquinol, it is likely that CybB can catalyse such a reaction when the menaquinone pool is reduced during respiration, equipping $E$. coli with a "superoxide gun" to attack competitors. The idea is especially attractive since periplasmic SOD is mainly expressed during stationary phase, while CybB transcription is enhanced during the logarithmic phase ${ }^{17}$. Finally, the forward reaction can be envisioned to happen under microaerobic almost anaerobic conditions where the $\mathrm{O}_{2} /$ superoxide ratio is low; like the environment $E$. coli faces as a commensal bacterium of the human gut. 


\section{References}

1 D'Autréaux, B. \& Toledano, M. B. ROS as signalling molecules: mechanisms that generate specificity in ROS homeostasis. Nature reviews. Molecular cell biology 8, 813-824, doi:10.1038/nrm2256 (2007).

2 Dröge, W. Free radicals in the physiological control of cell function. Physiological reviews 82, 47-95, doi:10.1152/physrev.00018.2001 (2002).

3 Pryde, K. R. \& Hirst, J. Superoxide is produced by the reduced flavin in mitochondrial complex I: a single, unified mechanism that applies during both forward and reverse electron transfer. J Biol Chem 286, 18056-18065, doi:10.1074/jbc.M110.186841 (2011).

4 Quinlan, C. L. et al. Mitochondrial complex II can generate reactive oxygen species at high rates in both the forward and reverse reactions. I Biol Chem 287, 27255-27264, doi:10.1074/jbc.M112.374629 (2012).

5 Dröse, S. \& Brandt, U. The Mechanism of Mitochondrial Superoxide Production by the Cytochrome bc1 Complex*. Journal of Biological Chemistry 283, 21649-21654, doi:https://doi.org/10.1074/ibc.M803236200 (2008).

6 Imlay James, A. \& Slauch James, M. Oxidative Stress. EcoSal Plus 3, doi:10.1128/ecosalplus.5.4.4 (2009).

7 Magnani, F. et al. Crystal structures and atomic model of NADPH oxidase. Proceedings of the National Academy of Sciences 114, 6764, doi:10.1073/pnas.1702293114 (2017).

8 Vignais, P. V. The superoxide-generating NADPH oxidase: structural aspects and activation mechanism. Cellular and molecular life sciences : CMLS 59, 1428-1459, doi:10.1007/s00018002-8520-9 (2002).

9 Nguyen, G. T., Green, E. R. \& Mecsas, J. Neutrophils to the ROScue: Mechanisms of NADPH Oxidase Activation and Bacterial Resistance. Frontiers in cellular and infection microbiology 7 , 373, doi:10.3389/fcimb.2017.00373 (2017).

10 Diaz, J. M. et al. Widespread Production of Extracellular Superoxide by Heterotrophic Bacteria. Science 340, 1223-1226, doi:doi:10.1126/science.1237331 (2013).

11 Huycke, M. M. et al. Extracellular superoxide production by Enterococcus faecalis requires demethylmenaquinone and is attenuated by functional terminal quinol oxidases. $\mathrm{Mol}$ Microbiol 42, 729-740, doi:10.1046/j.1365-2958.2001.02638.x (2001).

12 Korshunov, S. \& Imlay, J. A. Detection and quantification of superoxide formed within the periplasm of Escherichia coli. Journal of bacteriology 188, 6326-6334, doi:10.1128/jb.0055406 (2006).

13 Lin, P. C., Türk, K., Häse, C. C., Fritz, G. \& Steuber, J. Quinone reduction by the Nattranslocating NADH dehydrogenase promotes extracellular superoxide production in Vibrio cholerae. Journal of bacteriology 189, 3902-3908, doi:10.1128/jb.01651-06 (2007).

14 Fridovich, I. Superoxide dismutases. Annu Rev Biochem 44, 147-159, doi:10.1146/annurev.bi.44.070175.001051 (1975).

15 McCord, J. M. \& Fridovich, I. Superoxide Dismutase: AN ENZYMIC FUNCTION FOR ERYTHROCUPREIN (HEMOCUPREIN). Journal of Biological Chemistry 244, 6049-6055, doi:https://doi.org/10.1016/S0021-9258(18)63504-5 (1969).

16 Jenney Francis, E., Verhagen Marc, F. J. M., Cui, X. \& Adams Michael, W. W. Anaerobic Microbes: Oxygen Detoxification Without Superoxide Dismutase. Science 286, 306-309, doi:10.1126/science.286.5438.306 (1999).

17 Lundgren, C. A. K. et al. Scavenging of superoxide by a membrane-bound superoxide oxidase. Nature Chemical Biology 14, 788-793, doi:10.1038/s41589-018-0072-x (2018).

18 Winkler, J. R. \& Gray, H. B. Electron Flow through Metalloproteins. Chemical Reviews 114, 3369-3380, doi:10.1021/cr4004715 (2014).

19 Page, C. C., Moser, C. C., Chen, X. \& Dutton, P. L. Natural engineering principles of electron tunnelling in biological oxidation-reduction. Nature 402, 47-52, doi:10.1038/46972 (1999). 
20 Hayyan, M., Hashim, M. A. \& AlNashef, I. M. Superoxide Ion: Generation and Chemical Implications. Chemical Reviews 116, 3029-3085, doi:10.1021/acs.chemrev.5b00407 (2016).

21 Nishikimi, M., Appaji Rao, N. \& Yagi, K. The occurrence of superoxide anion in the reaction of reduced phenazine methosulfate and molecular oxygen. Biochemical and Biophysical Research Communications 46, 849-854, doi:https://doi.org/10.1016/S0006-291X(72)80218-3 (1972).

22 Peskin, A. V. \& Winterbourn, C. C. Assay of superoxide dismutase activity in a plate assay using WST-1. Free Radical Biology and Medicine 103, 188-191, doi:https://doi.org/10.1016/i.freeradbiomed.2016.12.033 (2017).

23 Abreu, I. A. \& Cabelli, D. E. Superoxide dismutases-a review of the metal-associated mechanistic variations. Biochimica et Biophysica Acta (BBA) - Proteins and Proteomics 1804, 263-274, doi:https://doi.org/10.1016/i.bbapap.2009.11.005 (2010).

24 Cerda, J. F. et al. Spectroelectrochemical measurements of redox proteins by using a simple UV/visible cell. Electrochemistry Communications 33, 76-79, doi:https://doi.org/10.1016/j.elecom.2013.05.004 (2013).

25 Trott, O. \& Olson, A. J. AutoDock Vina: Improving the speed and accuracy of docking with a new scoring function, efficient optimization, and multithreading. Journal of Computational Chemistry 31, 455-461, doi:https://doi.org/10.1002/jcc.21334 (2010).

26 Bielski, B. H. J., Cabelli, D. E., Arudi, R. L. \& Ross, A. B. Reactivity of HO2/O-2 Radicals in Aqueous Solution. Journal of Physical and Chemical Reference Data 14, 1041-1100, doi:10.1063/1.555739 (1985).

27 Nicholls, D. G. \& Ferguson, S. J. in Bioenergetics (Fourth Edition) (eds David G. Nicholls \& Stuart J. Ferguson) ix-x (Academic Press, 2013).

28 Alric, J., Pierre, Y., Picot, D., Lavergne, J. \& Rappaport, F. Spectral and redox characterization of the heme ci of the cytochrome b6f complex. Proc Natl Acad Sci U S A 102, 15860-15865, doi:10.1073/pnas.0508102102 (2005).

29 Murakami, H., Kita, K. \& Anraku, Y. Purification and properties of a diheme cytochrome b561 of the Escherichia coli respiratory chain. Journal of Biological Chemistry 261, 548-551, doi:https://doi.org/10.1016/S0021-9258(17)36126-4 (1986).

30 Fridovich, I. Quantitative aspects of the production of superoxide anion radical by milk xanthine oxidase. J Biol Chem 245, 4053-4057 (1970).

31 Porras, A. G., Olson, J. S. \& Palmer, G. The reaction of reduced xanthine oxidase with oxygen. Kinetics of peroxide and superoxide formation. Journal of Biological Chemistry 256, 90969103, doi:https://doi.org/10.1016/S0021-9258(19)52513-3 (1981).

32 Samoilova, R. I., Crofts, A. R. \& Dikanov, S. A. Reaction of Superoxide Radical with Quinone Molecules. The Journal of Physical Chemistry A 115, 11589-11593, doi:10.1021/jp204891n (2011).

33 Takahashi, M. \& Asada, K. Superoxide production in aprotic interior of chloroplast thylakoids. Archives of Biochemistry and Biophysics 267, 714-722, doi:https://doi.org/10.1016/00039861(88)90080-X (1988).

34 Husen, P. \& Solov'yov, I. A. Spontaneous Binding of Molecular Oxygen at the Qo-Site of the bc1 Complex Could Stimulate Superoxide Formation. Journal of the American Chemical Society 138, 12150-12158, doi:10.1021/jacs.6b04849 (2016).

35 Guo, J. \& Lemire, B. D. The Ubiquinone-binding Site of the Saccharomyces cerevisiae Succinate-Ubiquinone Oxidoreductase is a Source of Superoxide*. Journal of Biological Chemistry 278, 47629-47635, doi:https://doi.org/10.1074/ibc.M306312200 (2003).

36 Roginsky, V. A., Mohr, D. \& Stocker, R. Reduction of ubiquinone-1 by ascorbic acid is a catalytic and reversible process controlled by the concentration of molecular oxygen. Redox Report $\mathbf{2}$, 55-62, doi:10.1080/13510002.1996.11747027 (1996).

37 Getzoff, E. D. et al. Faster superoxide dismutase mutants designed by enhancing electrostatic guidance. Nature 358, 347-351, doi:10.1038/358347a0 (1992). 
38 Shin, D. S. et al. Superoxide Dismutase from the Eukaryotic Thermophile Alvinella pompejana: Structures, Stability, Mechanism, and Insights into Amyotrophic Lateral Sclerosis. Journal of Molecular Biology 385, 1534-1555, doi:https://doi.org/10.1016/i.jmb.2008.11.031 (2009). Sheng, Y. et al. Superoxide Dismutases and Superoxide Reductases. Chemical Reviews 114, 3854-3918, doi:10.1021/cr4005296 (2014).

40 Adam, V., Royant, A., Nivière, V., Molina-Heredia, F. P. \& Bourgeois, D. Structure of Superoxide Reductase Bound to Ferrocyanide and Active Site Expansion upon X-Ray-Induced PhotoReduction. Structure 12, 1729-1740, doi:https://doi.org/10.1016/i.str.2004.07.013 (2004).

41 Bedard, K. \& Krause, K. H. The NOX family of ROS-generating NADPH oxidases: physiology and pathophysiology. Physiological reviews 87, 245-313, doi:10.1152/physrev.00044.2005 (2007).

42 Sharma, P., Teixeira de Mattos, M. J., Hellingwerf, K. J. \& Bekker, M. On the function of the various quinone species in Escherichia coli. The FEBS Journal 279, 3364-3373, doi:https://doi.org/10.1111/j.1742-4658.2012.08608.x (2012).

43 Wissenbach, U., Kröger, A. \& Unden, G. The specific functions of menaquinone and demethylmenaquinone in anaerobic respiration with fumarate, dimethylsulfoxide, trimethylamine $\mathrm{N}$-oxide and nitrate by Escherichia coli. Archives of Microbiology 154, 60-66, doi:10.1007/BF00249179 (1990).

44 Tyson, K., Metheringham, R., Griffiths, L. \& Cole, J. Characterisation of Escherichia coli K-12 mutants defective in formate-dependent nitrite reduction: essential roles for hemN and the menFDBCE operon. Archives of Microbiology 168, 403-411, doi:10.1007/s002030050515 (1997).

45 Wallace, B. J. \& Young, I. G. Role of quinones in electron transport to oxygen and nitrate in Escherichia coli. Studies with a ubiA- menA- double quinone mutant. Biochimica et Biophysica Acta (BBA) - Bioenergetics 461, 84-100, doi:https://doi.org/10.1016/0005-2728(77)90071-8 (1977).

46 Ingledew, W. J. \& Poole, R. K. The respiratory chains of Escherichia coli. Microbiol Rev 48, 222271, doi:10.1128/mr.48.3.222-271.1984 (1984).

47 Imlay, J. A. Cellular Defenses against Superoxide and Hydrogen Peroxide. Annual Review of Biochemistry 77, 755-776, doi:10.1146/annurev.biochem.77.061606.161055 (2008).

48 Quick, K. L., Hardt, J. I. \& Dugan, L. L. Rapid microplate assay for superoxide scavenging efficiency. Journal of Neuroscience Methods 97, 139-144, doi:https://doi.org/10.1016/S01650270(00)00179-5 (2000).

49 Murakami, H., Kita, K. \& Anraku, Y. Cloning of cybB, the gene for cytochrome b561 of Escherichia coli K12. Molecular and General Genetics MGG 198, 1-6, doi:10.1007/BF00328692 (1984).

50 Jalukar, V., Kelley, P. M. \& Njus, D. Reaction of ascorbic acid with cytochrome b561. Concerted electron and proton transfer. Journal of Biological Chemistry 266, 6878-6882, doi:https://doi.org/10.1016/S0021-9258(20)89583-0 (1991).

51 Schultz, B. E., Edmondson, D. E. \& Chan, S. I. Reaction of Escherichia coli Cytochrome bo3 with Substoichiometric Ubiquinol-2: A Freeze-Quench Electron Paramagnetic Resonance Investigation. Biochemistry 37, 4160-4168, doi:10.1021/bi971714y (1998).

52 Magalon, A. et al. Heme Axial Ligation by the Highly Conserved His Residues in Helix II of Cytochrome b (Narl) of Escherichia coliNitrate Reductase A (NarGHI)*. Journal of Biological Chemistry 272, 25652-25658, doi:https://doi.org/10.1074/ibc.272.41.25652 (1997).

53 Takeuchi, F., Hori, H., Obayashi, E., Shiro, Y. \& Tsubaki, M. Properties of Two Distinct Heme Centers of Cytochrome b561 from Bovine Chromaffin Vesicles Studied by EPR, Resonance Raman, and Ascorbate Reduction Assay. The Journal of Biochemistry 135, 53-64, doi:10.1093/jb/mvh006 (2004).

54 Oakhill, J. S., Marritt, S. J., Gareta, E. G., Cammack, R. \& McKie, A. T. Functional characterization of human duodenal cytochrome $b$ (Cybrd1): Redox properties in relation to 
iron and ascorbate metabolism. Biochimica et Biophysica Acta (BBA) - Bioenergetics 1777, 260-268, doi:https://doi.org/10.1016/j.bbabio.2007.12.001 (2008).

55 Iyanagi, T., Yamazaki, I. \& Anan, K. F. One-electron oxidation-reduction properties of ascorbic acid. Biochimica et Biophysica Acta (BBA) - Bioenergetics 806, 255-261, doi:https://doi.org/10.1016/0005-2728(85)90103-3 (1985).

56 Asard, H., Barbaro, R., Trost, P. \& Bérczi, A. Cytochromes b561: Ascorbate-Mediated TransMembrane Electron Transport. Antioxidants \& Redox Signaling 19, 1026-1035, doi:10.1089/ars.2012.5065 (2012).

57 Smirnova, I. A., Hägerhäll, C., Konstantinov, A. A. \& Hederstedt, L. HOQNO interaction with cytochrome $b$ in succinate:menaquinone oxidoreductase from Bacillus subtilis. FEBS Letters 359, 23-26, doi:https://doi.org/10.1016/0014-5793(94)01442-4 (1995).

58 Yap, L. L. et al. The quinone-binding sites of the cytochrome bo3 ubiquinol oxidase from Escherichia coli. Biochimica et Biophysica Acta (BBA) - Bioenergetics 1797, 1924-1932, doi:https://doi.org/10.1016/j.bbabio.2010.04.011 (2010).

59 Ma, J., Puustinen, A., Wikström, M. \& Gennis, R. B. Tryptophan-136 in Subunit II of Cytochrome bo3 from Escherichia coli May Participate in the Binding of Ubiquinol. Biochemistry 37, 1180611811, doi:10.1021/bi9809977 (1998).

60 Murai, M. et al. Characterization of the Ubiquinone Binding Site in the Alternative NADHQuinone Oxidoreductase of Saccharomyces cerevisiae by Photoaffinity Labeling. Biochemistry 49, 2973-2980, doi:10.1021/bi100005j (2010).

61 Blaza, J. N. et al. The mechanism of catalysis by type-II NADH:quinone oxidoreductases. Scientific Reports 7, 40165, doi:10.1038/srep40165 (2017).

62 Anderson, R. F. et al. Electron-Transfer Pathways in the Heme and Quinone-Binding Domain of Complex II (Succinate Dehydrogenase). Biochemistry 53, 1637-1646, doi:10.1021/bi401630m (2014). 